\title{
Fallgeschichte, Historia, Klassifikation
}

\section{François Boissier de Sauvages bei der Schreibarbeit}

\author{
Volker Hess und J. Andrew Mendelsohn
}

Case History, Historia, Classification. François Boissier de Sauvages at Work on Paper

\begin{abstract}
What was classification as it first took modern form in the eighteenth century, how did it work, and how did it relate to earlier describing and ordering? We offer new answers to these questions by considering an example less well known than that of botany or zoology, namely medicine, and by reconstructing practice on paper. The first and bestknown disease classification is the "nosology" of the Montpellier physician François Boissier de Sauvages de Lacroix. Its several editions, we show, were less products than process: published tools for building a classification system. The disorder of a hitherto unstudied notebook that Boissier de Sauvages kept throughout this process provided a way of breaking with the topical order of earlier physicians' humanistic commonplace books of disease observation while sustaining the paper practices those earlier physicians—and Sauvages himself as a student-had used to order disease. This suggests a different picture of historical change than that of a scholarly world of ordered words giving way to a scientific one of ordered things. Classification, in the case of Sauvages' nosology, arose through an incomplete break with, and intensified practice of, a past way of ordering the described world. The humanist paper practice that had made observationes, differently applied, now made species. Classification into genera and species by similarity and difference, which Sauvages' nosology shared with botany, was an algorithm of paper and ink practice - in its operation more machine-like than humanist textual practice yet in its effects more creative and re-creative of categories and questions of relationship. Thus a new empiricism of generalizations (species) arose out of the older, Renaissance empiricism of particulars (observations, facts).
\end{abstract}

Keywords: Nosology, eighteenth-century medicine, humanist textual method, observatio, disease species

Schlüsse/wörter: Nosologie, Medizin des 18. Jahrhunderts, humanistische Methode, Observatio, Krankheitsspezies

In ihrem groß angelegten Panorama des frühneuzeitlichen Frankreichs zeichnen Laurence Brockliss und Colin Jones kein schmeichelhaftes Bild der medizinischen Wissenschaft. Obwohl sie den Aufbruch in ein neues wissenschaftliches Zeitalter beschworen, seien sich Ärzte und Gelehrte weder über die Mittel noch Methoden des wissenschaftlichen Erkenntnisgewinns einig gewesen. Man habe zwar Beobachtung, Erfahrung und Versuche - mit einem Wort: expérience - gefordert, ohne jedoch Kriterien für deren Qualität oder 
Umfang anzugeben. Vielmehr sei man am Krankenbett weitgehend im Dunkeln umher getappt, zumal man in diesen vorstatistischen Zeiten keine verbindliche Vorstellung darüber besessen habe, wie man Beobachtungen sammeln, zusammenstellen und bewerten solle. Diese Diskrepanz zwischen dem rhetorischen Anspruch der neuen Wissenschaften und ihrer empirischen Praxis zeige besonders augenfällig das Notizbuch von François Boissier de Sauvages de la Croix (1706-1767). Dieser „vollkommene und entzückend unordentliche Mischmasch" vereine eine wilde Sammlung von Krankengeschichten aus der Praxis, Fallbeschreibungen aus dem Hospital, Feldbeobachtungen, Sektionsberichten, aber auch Anmerkungen über das Gedeihen seiner Kinder und Katzen sowie Notizen von Selbstversuchen. Folgt man diesem Hinweis lässt sich diese Aufzählung um weitere Genres eines neuen Empirismus erweitern, nämlich um Versuchsprotokolle, physiologische Erörterungen, Textauszüge aus der medizinischen Literatur, Konzepte und Versatzstücke späterer Publikationen. Dieses „potpourri“ stehe, um noch einmal das Standardwerk für die Geschichte der frühneuzeitlichen Medizin anzuführen, für die Ungestalt und Inkohärenz der zeitgenössischen „,research methodologies" (Brockliss/Jones 1997: 672).

Spätestens an diesem Punkt kommt der Leser ins Stutzen. Der erste Grund ist offensichtlich: Die moderne Medizin zählt Sauvages zu den Heroen ihrer Geschichte und feiert den Verfasser der 1731 erstmals publizierten Nouvelles classes de maladies als Begründer der medizinischen Nosologie und damit jenes Unternehmens, das dem Denken und Handeln der Ärzte verbindliche Begriffe und Kategorien vorgibt - heute in Form der International Classification of Diseases (ICD) oder des Diagnostic Statistical Manuals (DSM) (Pichot 1994, Foucar 2001, Lenfant/Loeb/Peuvrel/Sauvage 2001). Die systematische Klassifikation gilt in der Medizin wie in anderen Bereichen als Inbegriff von Ordnung. Sie weist jeder Krankheit wie in einem gut aufgeräumten Haushalt einen definierten Platz zu. Selbst neue Syndrome oder pathologische Befunde finden - über kurz oder lang - bei einer der nächsten Revisionen Eingang in die Systematik (Cooper 2004, Mayers/Horwitz 2005) oder fallen aus dem Kanon (Bowker/Star 1999). Und der Anfang aller klinischer Ordnung soll - so muss man angesichts von Sauvages' Notizbuch fragen - ein solches Potpourri sein?

Nun ließe sich einwenden, dass jeder Ordnung - wie im besagten Haushalt - ein Zustand der Unordnung vorausgeht. Doch genau diese Alltagserfahrung gibt Anlass für weiteres Erstaunen. Folgt man den Hinweisen von Brockliss/Jones 1997, so befinden sich in den Archives départementales de l'Hérault nicht nur das im Findbuch als Livre de raison (im Folgenden: Livre) aufgeführte Notizbuch. ${ }^{1}$ Vielmehr wird in Montpellier auch ein von der Forschung bislang noch nicht berücksichtigtes Manuskript verwahrt, das ebenfalls Sauvages zugerechnet wird. Dieses im Findbuch als Matière médicale (im Folgenden: Matière) bezeichnete Konvolut ${ }^{2}$ ist in den Studienjahren 
Sauvages' (1722-1726) entstanden - und lässt jede Unordnung vermissen. Ganz im Gegenteil stehen diese Aufschriebe für eine humanistische Ordnung des Wissens, wie wir im ersten Teil unseres Beitrages zeigen werden.

Einen dritten Vorbehalt gegen die Annahme einer mangelhaften Forschungsmethodologie, sofern man diesen anachronistischen Begriff bemühen möchte, liefert schließlich die Chronologie. Die ersten Eintragungen im Livre datieren vom Mai 1745 - also nach dem Erscheinen der ersten Auflagen der Nosologie von Sauvages. ${ }^{3}$ Und als die letzten im April 1765 vorgenommen wurden, hatte Sauvages bereits die fünfte Auflage seiner Klassifikation publiziert. Das Durcheinander ging damit nicht der Ordnung voraus. Vielmehr begleitete es über die ganze Zeit die Arbeit am nosologischen System (King 1966, Martin 1990). Die Nouvelles classes wurden 1739 als Pathologica methodica neu aufgelegt, 1752 folgte die zweite und 1759 die dritte Auflage. Deren überarbeitete Fassung kam schließlich als Nosologie méthodique (1762) respektive Nosologia methodica (1763) auf den Markt, 1768 folgte posthum in Latein sowie 1772 auf Französisch die Auflage letzter Hand. Angesichts der ausgefeilten Ordnung der handschriftlichen Arbeiten am naturhistorischen System $^{4}$ drängt sich folglich die Frage auf: Kommt der Koinzidenz von Ordnung und Unordnung eine systematische Funktion zu? War sie Ausdruck, wie der Blick auf die studentischen Mitschriften nahelegt, einer Arbeitstechnik, die sich dem ordentlichen Schreiben in humanistischer Tradition verweigerte? Wie verhielten sich die Abgrenzung benennbarer Krankheitsarten und ihre systematische Anordnung zum humanistischen Sammeln von Lesefrüchten nach „Orten“ oder Gemeinplätzen (Loci communes)? Und war die unordentliche Buchführung womöglich Teil einer Arbeitstechnik, ${ }^{5}$ die der jahrzehntelangen Arbeit am nosologischen System zugrunde gelegen hat?

Das unordentliche Schreiben hatte, so unsere These, in der Tat System. Es verweist auf eine Transformation der Loci communes in eine medizinische Klassifikation, mit der das epistemische Genre der Observatio in eine Historia morbi überführt wurde. Bevor wir dieses Argument entfalten, ist es erforderlich, die bereits erwähnten Quellengattungen etwas ausführlicher vorzustellen. Im ersten Teil unseres Beitrags werden wir die Aufzeichnungen am Krankenbett aus der klinischen Ausbildung Sauvages' beschreiben und ihre Funktion als paper technology (te Heesen 2005b) diskutieren. Unter diesem Sammelbegriff verstehen wir die Summe aller Schreibverfahren (wie Listen oder Formulare), Text- (wie Exzerpt oder Index) und Papiertechniken (wie Karteikarten oder Bandakten) und der damit verbundenen Werkzeuge (wie Stifte, Klebstoff oder Schere), die (intendiert und unbeabsichtigt) beim Festhalten, Sammeln, Akkumulieren von (direkten oder vermittelten) Beobachtungen eingesetzt wurden (Hess/Mendelsohn 2010). In den folgenden Abschnitten gehen wir auf die Ordnungsstruktur der Nouvelles classes und deren Bezug zum Livre ein. Nur selten findet man in der Literatur der Frühen Neuzeit die handschriftlichen Vorarbeiten für das daraus hervorgegangene 
Druckwerk. Diesen Glücksfall wollen wir nutzen, um beide Textformen Notizbuch und gedruckten Klassifikationsschlüssel („Cléf de classes“ (Sauvages 1772. Bd. 1: 321-324)) - als zwei komplementäre Elemente eines gemeinsamen Aufschreibesystems zu begreifen. ${ }^{6}$ Zunächst werden wir den methodischen Ansatz der Systematik vorstellen, dann exemplarisch die verallgemeinernde Technik des Exzerpierens diskutieren und schließlich das Schärfen der kategorialen Begriffe bei der Arbeit am System verfolgen. Die historische Rekonstruktion der wechselseitigen Verschränkung von Notizbuch und Druckwerk und ihrer sich gegenseitig ergänzenden Papiertechniken erlaubt uns, die Herausbildung der neuen Gattung der Krankheitsklassifikation (Nosologie) „in the making“ (Johns 1998) zu analysieren - und damit zugleich einen Beitrag zum Verständnis der systematischen Klassifikation im 18. Jahrhundert zu geben. Wodurch unterscheidet sich die naturhistorische Klassifikation von der humanistischen Ordnung des Wissens? In welchem Verhältnis stehen die zugrundeliegenden Aufschreibeverfahren und Papiertechniken zu den humanistischen Texttechniken des Sammelns, Exzerpierens und Ordnens? Und in welcher Weise veränderte dieses Wissensmanagement (Zedelmaier 2009) die humanistische Ordnung der Natur?

\section{Fallgeschichten in humanistischer Schreibarbeit}

Wie im frühen 18. Jahrhundert Wissen zusammengetragen und systematisiert wurde, lässt sich am Beispiel der von Sauvages hinterlassenen Mitschriften aus seiner Studienzeit anschaulich demonstrieren. Das Konvolut besteht aus mehreren, nachträglich zusammengebundenen Heften. Ein Heft gibt eine Übersicht über die von Jacques Lazerme (1676-1756) gelehrte Matière médicale, ein weiteres umfasst einen, leider nur rudimentären Auszug von Antoine Deidiers Compendium morborum abdominis, daneben finden sich Mitschriften von leçons sowie Lazermes Interpretation eines Aphorismus des divi Hippocratis (datiert 1724). Ein weiteres, 80 Seiten umfassendes Heft enthält ausschließlich Krankenbeobachtungen, womit es dem medizinischen Genre des casebook entspricht. ${ }^{7}$ Die meisten Hefte werden mit einem als Tabla überschriebenen Register eingeleitet, das die unter bestimmten Schlagwörtern notierten Aufzeichnungen erschließt. Diese Technik, das Aufschreiben nach Loci, Tituli, oder Capita zu strukturieren und durch einen Index zu verwalten, war Teil jener Texttechnik (Methodus), die zeitgenössisch in Grammatikschulen und Universitäten gelehrt und trainiert wurde (Waquet 2000, Campi/ De Angelis/Goeing 2008). Sie weist Sauvages' Studienaufzeichnungen als Loci communes aus (Stolberg 2013). Diese humanistische Aufschreibetechnik wurde nicht nur in der Bibliothek eingesetzt, um Ordnung in die Lesefrüchte zu bringen (Zedelmaier 1992, Meinel 1995), sondern auch für die Notizen aus 
Vorlesungen oder für die Sammlung von Beobachtungen herangezogen (Blair 2010b). Aufschriebe in Form der Loci communes bildeten die verbindliche Methode des Wissenserwerbs. Als Grundlage der zeitgenössischen Wissenschaft stellt diese Technik auch die Funktion jener von Brockliss und Jones (1997: 672) so schmerzlich vermissten Forschungsmethodologie dar.

In die Loci communes gingen auch Krankenbeobachtungen ein, wie Sauvages' Fallsammlung anschaulich illustriert. Entstanden sind seine Berichte zwischen Anfang 1727 und Mitte 1728, vermutlich am Hôpital Saint-Eloi, dem größten der drei Hospitäler Montpelliers (Jones 1982, 1989). Zu diesem Zeitpunkt hatte Sauvages das Studium bereits abgeschlossen (Grasset 1896: 23) und ging dem das Krankenhaus betreuenden Antoine Deidier (1670-1746) offenbar als persönlicher Assistent (médecin adjoint) zur Hand, eine Aufgabe, die dann ab 1732 regelmäßig als Amt (en titre) vergeben wurde. ${ }^{8}$

Auch wenn die gesammelten Fallberichte nicht explizit so ausgewiesen sind, so belegt die Angabe der Bettnummer oder der Umstände, unter denen die Behandelten ins Krankenhaus aufgenommen wurden, doch die Herkunft der Beobachtungen aus dem Hospital. Zudem sind immer wieder Anweisungen und Erläuterungen von „M. Deidier“ vermerkt, aus denen eine Ausbildungssituation hervorgeht. Fallsammlungen wurden oft aus Praxistagebüchern („excerptae ex diario practico“) publiziert (Stolberg 2007: 84). Sauvages' Aufzeichnungen hingegen stellen eines der frühesten Beispiele für handschriftliche Notate aus einem klinischen Ausbildungszusammenhang dar. Die Art und Weise der Aufschriebe gingen dem Modell prägenden Beispiel der Leidener Klinik zeitlich voran (Risse 1989). Denn Sauvages nahm in jenen Jahren, als er sich - vor seinem Ruf an die Fakultät von Montpellier intensiv um ein öffentliches Amt bemühte, ${ }^{9}$ offenbar die Funktion eines Interns („médecin adjoint en titre“) wahr. Diese Ausbildung am Krankenbett fand somit, im Gegensatz zur Mitarbeit in der Praxis eines älteren Kollegen (Pomata 2010) oder zum Collegium clinicum (Brockliss/Jones 1997: 192-198), im Hospital statt. Dieses Modell wurde in Leiden systematisch erprobt und schließlich unter Herman Boerhaave (Risse 1989, Beukers 1989: 147) in den späten 1720er Jahren institutionalisiert und über seine Schüler (Probst 1972, Karenberg 1997) verbreitet. ${ }^{10}$

Auf jeden Fall lässt sich an den klinischen Aufzeichnungen die damals übliche gelehrte Schreibpraxis veranschaulichen. Denn Sauvages' Schreibheft gibt die Fallgeschichten nicht als Journal (Hess 2010: 325) in der zeitlichen Folge ihrer Beobachtung wieder. Wurden auf Seite 19 unter dem Titel „fievres continuë“ von „Alexandre le Medecin et Mr Freind après Galien“ Lesefunde aus der Literatur zusammengetragen, so folgt auf der nächsten Seite mit Datum vom 16. Mai 1727 eine Fallgeschichte eines an diesem Fieber erkrankten Patienten mit der Angabe seiner Behandlung „hier et aujourdhui“. 11 Die Fallgeschichte eines kohlschwarzen Rotlaufs („eresipele charbonneux") am Ende des Heftes wurde hingegen bereits am 28. Februar 
1727 aufgeschrieben. ${ }^{12}$ Dass die Beobachtungen zeitnah eingetragen wurden, macht ihr unmittelbarer Stil deutlich: ,il est mort hier et aujourd'hui samedi on le disseque à l'université “ ${ }^{13}$ Gelegentlich ist solchen Passagen nachträglich noch ein Datum eingefügt worden, so als ob Sauvages im letzten Moment noch aufgefallen war, dass „heute" keine verlässliche Zeitangabe darstellt. ${ }^{14}$

Nicht die chronologische Folge der Fallgeschichten, sondern die Ergänzung der vorgeschriebenen Loci communes bildete die Leitmaxime dieser Aufzeichnungspraxis. Das vorangestellte Register verdeutlicht die Technik, mit der die Loci communes angefertigt wurden: Sauvages schrieb auf der ersten Seite - von 1 bis 80 - alle Seitenzahlen vor. Jede dritte bis fünfte betitelte er vorab mit einer Krankheitsbezeichnung, zu der dann auf den entsprechend freigehaltenen Seiten die Beobachtungen vermerkt wurden - manche länger, im Ausnahmefall sogar über drei Seiten, andere kürzer. So findet sich unter dem Eintrag des Rotlaufs („eresipele“) sowohl die bereits erwähnte kohlschwarze Ausprägung („eresipele charbonneux“) als auch, durch einen Querstrich getrennt, der Eintrag für einen Patienten in Bett 15 (,la 15e a un eresipele aux jouës") (vergleiche den dritten Textabschnitt in Abb. 1).

Sauvages' casebook entspricht somit dem systematischen Typus der Loci communes (Stolberg 2013). So folgen die auf der ersten Innenseite des Heftes vorgeschrieben tituli oder loci einer groben pathologischen Ordnung (zuerst Entzündungen, dann Fieber, Ausleerungen, Geisteskrankheiten, Schmerzzustände und schließlich Auszehrungen), nach der dann auf der indexierten Seite die Fallbeschreibungen vorgenommen wurden. Für manche pathologischen Zustände konnte Sauvages zwei oder gar drei Beobachtungen vermerken. Andere Loci konnte Sauvages während seiner Zeit im Hospital nicht füllen. So finden sich auch in den Matières gelegentlich unbeschriebene Seiten. ${ }^{15}$ Solche Leerstellen suchte Sauvages offensichtlich zu vermeiden, indem er das herkömmliche System der Loci communes mit einer sequentiellen Aufzeichnungstechnik kombinierte. ${ }^{16}$ So schrieb Sauvages auf manchen Seiten Fallgeschichten auf, die erkennbar erst nachträglich in die Ordnung eingefügt und im Register der Loci indexiert wurden, wobei die ursprüngliche Ordnung nicht immer eingehalten wurde. ${ }^{17}$ Diese Form des Schreibens verfolgt also nicht die für ein Krankenjournal übliche chronologische Aufzeichnung (Hess/Schlegelmilch 2013). Ihr Ziel ist vielmehr eine Sammlung von Observationes nach dem Raster vorgegebener Loci communes. Sauvages' casebook reiht sich damit ein in das Unternehmen des 17. Jahrhunderts, mit dem die Observatio zu einem neuen literarisch-medizinischen Genre aufstieg (Stolberg 2007, Pomata 2011).

Wir wissen nun, dass die Unordnung des Livre nicht darauf zurückgeführt werden kann, dass Sauvages nicht ordentlich aufschreiben konnte. Ganz im Gegenteil war er - wie vermutlich viele Gelehrte seiner Zeit - in den humanistischen Schreib- und Aufzeichnungstechniken ausgebildet worden und 


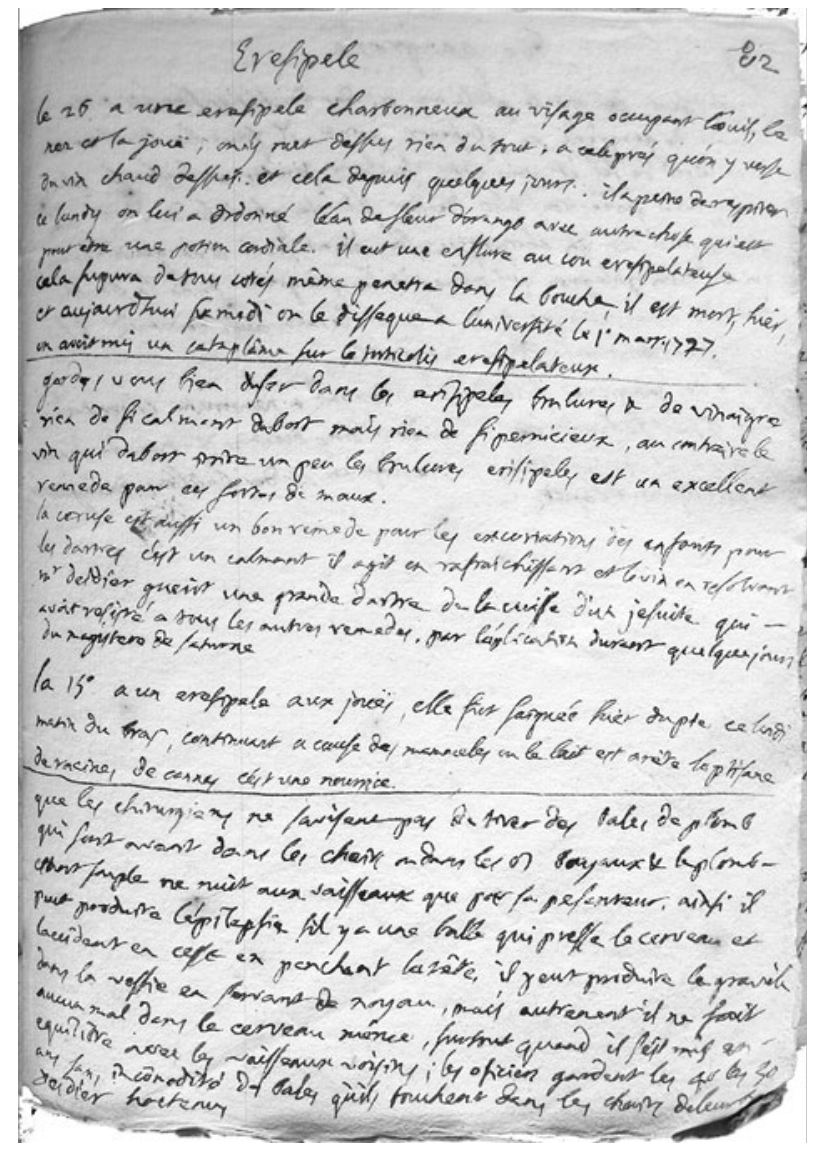

Abb. 1 Sauvages Aufzeichnungen zum Rotlauf („,eresipele“) im Matière: 82 (Mit freundlicher Genehmigung des Archives Départementales de I'Hérault). ${ }^{18}$

beherrschte, wie die Studienhefte eindrücklich belegen, sehr wohl die mit den Loci communes verbundenen Techniken des Exzerpierens und Indexierens.

\section{Von der Anfertigung der Loci communes zu einer Methodus systematica}

Sauvages wusste, was er kritisierte als er 1731 weitgehend anonymisiert $\left(\mathrm{S}^{* * * * *}\right.$ de $\left.{ }^{* * *}\right)$ die Nouvelles classes de maladies publizierte (Martin 1990), die ihn drei Jahre später auf den Lehrstuhl der Fakultät in Montpellier verhalfen. Das knapp 450-seitige Werk verwirft nämlich die Aufschreibetechnik der Loci, allerdings weder das Exzerpieren noch das Sammeln, sondern die zeitgenössische, in „Büchern, Überschriften, Artikeln und Paragrafen“ (1772, Bd. 1: 97) 
ausgeführte Anordnung der Krankheitsbeschreibungen. Diese Ablehnung veranschaulichte und begründete Sauvages in den folgenden Auflagen mit vielen Beispielen: Eine alphabetische Anordnung bringe Erkrankungen zusammen, die nicht zusammengehören wie Apoplexie (Schlaganfall) und Alopezie (Haarausfall) oder Paralyse (Lähmung) und Paronychie (Nagelbettenzündung), reiße hingegen zusammengehörige Krankheiten auseinander wie Arthritis und Rheumatismus (1763, Bd. 1: 26). Die anatomische Methode sei hingegen konfus und bleibe im ,jargon des Scholastiques“ stecken (1772, Bd. 1: 105). Wer wolle beispielsweise beim Lebenden die Grenzen zwischen einer Erkrankung der Milz und einer Krankheit der Leber, zwischen einer des Dünnund einer des Dickdarms unterscheiden, wenn dieser Unterschied selbst bei der Leichenöffnung kaum zu finden sei. Trügerisch seien schließlich alle Versuche, Krankheiten nach Qualitäten, Ursachen oder ätiologischen Mechanismen zu unterscheiden, über die es keine Einigkeit zwischen den Schulen gäbe. Die Angabe der Loci, die Definition der Klassen, Gattungen und Arten müsse „klar und kurz“ sein, weshalb sie nicht auf „hypothetischen, rein pathologischen oder gar imaginären Ursachen [basieren dürften] wie bei den Alten überliefert oder bei den Deutschen [im Original: les Allemands] noch praktiziert" (1731: xvii). ${ }^{19}$

Als Alternative schlägt Sauvages eine Methodus systematica vergleichbar jener der Botaniker (,semblable à celui des Botanistes“ (ebd.: Titel)) vor. Seine explizite Anleihe bei Joseph Pitton de Tournefort (1656-1708), dessen Werk Sauvages während eines einjährigen Aufenthaltes in Paris studiert hatte, darf aber nicht vorschnell mit einem ontologischen oder substantiellen Krankheitsverständnis gleichgesetzt werden, demzufolge Krankheiten als Gegenstände der Natur betrachtet und klassifiziert werden dürfen (vergleiche Faber 1930, Berghoff 1947, King 1966, Williams 2003). Auch wenn Sauvages der Überzeugung war, gleich den Botanikern Arten, Gattungen und Klassen zu unterscheiden, so erzeugte dieses Vorgehen „im Geiste Sydenhams“ (1763: Titel, Hess 1993: 34-43) im medizinischen Aufschreibesystem andere Effekte als in der Verzeichnung von Lesefrüchten und Beobachtungen aus der Pflanzenwelt (Müller-Wille/Scharf 2011), auf die wir im letzten Abschnitt unseres Beitrages noch einmal eingehen werden.

An dieser Stelle ist zunächst festzuhalten, dass Sauvages mit diesem prominenten Verweis nicht auf den Objektstatus der botanischen Sammlungsgegenstände abhob, sondern auf ein methodisches Verfahren. Die zeitgenössischen Naturforscher wie Sauvages begriffen unter methodus die „richtige Lern- und Lehrmethode“ (recta discendi E docendi methodus), die aller Wissenschaft (unica mater scientiae) (1739: 94) zugrunde liege. Den entscheidenden Unterschied zur Ordnung der Loci communes bildete also nicht das Botanische der Methode, sondern das Methodische eines Verfahrens, das auch in der Botanik verwendet wurde. Diese Methode zeichnete sich in zweierlei Hinsicht aus: erstens durch ihren kategorialen Aufbau (wie Art 
und Gattung) und zweitens durch die logische Struktur der Klassenbildung. Jeder Locus, jede "Systemstelle“ war durch die gemeinsamen Merkmale (=Bezeichnung der Gattung) definiert, die sie subsumiert, und zugleich durch jene Merkmale, durch die sie sich von allen anderen (das Art-Epitheton) unterscheidet. So ist beispielsweise allen Fieberkrankheiten eine Erhöhung des Pulsschlags und eine Beeinträchtigung jener Lebenstätigkeiten gemein, die sich in Schüttelfrost, Hitze und Frost, Schwäche, Übelkeit und Leib- und Kopfschmerzen äußert. Die Regelmäßigkeit und Beständigkeit der Pulserhöhung unterscheiden wiederum die Klasse der Fieber von allen anderen, insbesondere von den entzündlichen Erkrankungen, bei denen die erhöhte Pulsfrequenz „symptomatisch, akzidentell, zweitrangig und unbeständig“ bleibe (1731: 33 f.). Beide Elemente der Methode sind zwar interdependent, da die Bezeichnungen der Art nicht frei mit denen der Gattung kombinierbar sind. Sauvages entwickelte die kategoriale Struktur aber nicht aus der Logik der Methode. Er baute sein System auch nicht, wie von Bacon programmatisch gefordert, Schritt für Schritt auf den Differenzen des Partikulären auf. Stattdessen fasste er die Krankheitsarten erst zu Klassen (nouvelles classes) zusammen, um dann rekursiv die mittleren Kategorien der Ordnungen und Gattungen herauszuarbeiten (Martin 1990).

Damit schufen die Nouvelles classes die Grundlage einer systematischen Ordnung. Auf knapp 400 der 450 Seiten präsentiert das in kleingedruckter Schrift publizierte Werk eine nicht enden wollende Auflistung aller Krankheiten. Insgesamt 2.400 Krankheitsarten wurden zu Gattungen, Ordnungen und Klassen gruppiert, deren Definition, nämlich die unterscheidenden Merkmale, Sauvages kurz beschrieb. Damit gleicht die Schrift jener Form des Registers, das Sauvages als Intern führte - und unterscheidet sich zugleich von diesem dadurch, dass es keine zusammengetragenen Observationes erschloss, sondern die zukünftige Papierarbeit gewissermaßen vorbereitend strukturierte. Die 1739 in Latein veröffentlichte erste und 1752 zweite Auflage der Pathologia methodica brachen mit der Beschreibung des Klassifikationsverfahrens bei den Gattungen - ohne Auflistung der Spezies - ab. Sie erörterten die pathologischen Einteilungsprinzipien, mit der Sauvages die systematische oder - wie es ab 1763 hieß - nosologische Methode begründete. Denn 1759 führte er wieder eine Auflistung der Spezies jeder Gattung ein, allerdings nicht, wie in den Nouvelles classes in Form einer Definition, sondern auf eine bloße Liste verkürzt (vgl. Abb. 2). In der Nosologia von 1762 wurde dieser Index schließlich gefüllt und jede Spezies eingehend beschrieben, womit sich die einstmals schmale Schrift zu einem mehrbändigen Werk ausweitete. Die verschiedenen Auflagen der Nouvelles classes entwickelten folglich einen Index, der zum einen gleich den humanistischen Tituli das Sammeln vorantrieb, zum anderen - in seiner beständigen Weiterentwicklung - dieses Sammeln abbildete. 


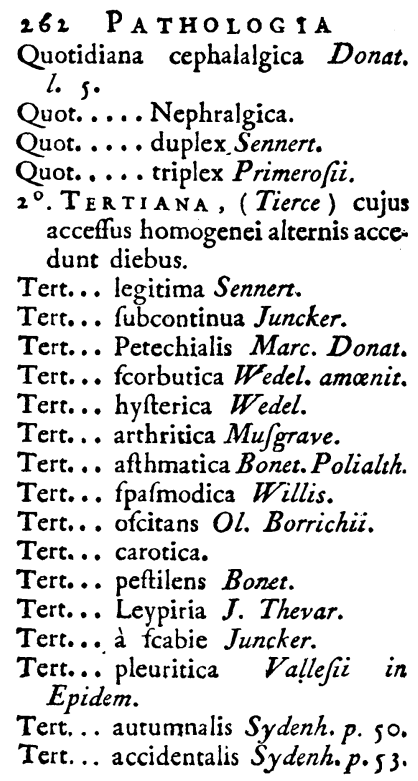

Abb. 2 Auflistung der Febres intermittentes quotidiana et tertiana in Sauvages 1759: 262.

Dass Sauvages sich seit seiner Berufung nach Montpellier darum bemühte, die Fächer des Registers zu füllen, belegt das Livre. Viele Einträge von dort finden sich wörtlich oder fast wörtlich in der Nosologia wieder wie die Beschreibung der überimpften Pocken (Variola inoculata). ${ }^{20}$ So exzerpierte Sauvages beispielsweise im Herbst 1761 eine Beobachtung von Johann Friedrich Meckel dem Älteren (1724-1774), die drei Jahre zuvor in den Memoires der Berliner Akademie als Inflammatio cordis $^{21}$ veröffentlicht worden war und nun als Carditis spontanea zwei Jahre später in die Nosologie (1763, Bd. 1: 482) eingereiht wurde. ${ }^{22}$ Offensichtlich durchforstete Sauvages in guter, humanistischer Manier alle ihm zugänglichen Veröffentlichungen nach brauchbaren Observationes. Neben Journalen wie der Histoire de l'Académie Royale des Sciences et des Belles-Lettres de Berlin oder dem Recueil pérodique d'Observations de Médecine, Chirurgie, Pharmacie exzerpierte Sauvages auch Reiseberichte und andere naturkundliche Genres. So findet sich beispielsweise ein Auszug aus Linnés Flora Lapponica (Linné 1737), mit dem Sauvages die einzige medizinische Observatio aus einer Seiten füllenden Darstellung des Echten Engelwurz (Angelica foliorum impari lobato) isolierte und in seine Aufzeichnungen übertrug. ${ }^{23}$ Bei dieser Schreibarbeit erwies sich Sauvages als guter Schüler und humanistischer Arzt.

Doch Sauvages exzerpierte keineswegs nur für die Erweiterung seiner medizinischen Artensammlung. Während Carl Linné (1707-1778) bei jeder Revision nicht nur im übertragenen Sinne „Leerblätter“ für die Beschreibung neuer Arten in sein Systema naturae einschob, brach Sauvages die im Index 
fixierte Ordnungsstruktur der Nouvelles classes bei jeder Neuauflage erneut auf - aber nicht, um seine Sammlung neu zu sortieren. Vielmehr nahm jede Überarbeitung eine Neudefinition kategorialer Abteilungen vor, wie dieses Beispiel zeigt: Zwischen Februar und Oktober 1748 exzerpierte Sauvages die Reisebeschreibungen Linnés. ${ }^{24}$ Er übertrug dessen Observatio einer bei den Samen verbreiteten Kolik unter Angabe der Fundstelle („Linnae Fl Lappon pag $\left.69^{\prime \prime}\right)$ in sein Notizbuch. Dabei kam es allerdings zu einer ersten Verschiebung. Hatte Linné vermutet, dass die als Ullem oder Hotme bezeichnete Krankheit der spastischen Kolik nach Scheuchzer entspreche, ${ }^{25}$ so übernahm Sauvages diese Einordnung der Erkrankung nicht. Noch im Aufschreiben strich er das Epitheton „spasmodica“ durch und ersetzte es durch „lapponica“. Die scheinbar flüchtige Korrektur sollte nicht unterschätzt werden. Erstens kreierte bereits die im Zuge des Exzerpierens und Umtragens geschaffene Kombination aus Gattung (Colica) und Epitheton (lapponica) eine neue Spezies. Zweitens generierte das neue Epitheton lapponica zugleich, getreu dem Verfahren der méthode systematique, eine Differenz. Unmittelbar an die im Exzerpt vorgenommene Absetzung von Linnés Zuordnung findet sich die Anmerkung „Colica spasmodica Scheuchzeri?“26 Das Fragezeichen markiert den produktiven Impuls dieser Aufschreibetechnik. 15 Jahre später listet die Nosologia methodica beide Spezies auf, allerdings in unterschiedlichen Klassen.

Das Fragezeichen war damit aufgelöst. Während Linné das obskure Ullem noch als lokale Variante der von Johann Jakob Scheuchzer (1672-1733) beobachteten Kolik von Bergbewohnern (Scheuchzer 1723: 14) betrachtete, hatte Sauvages zwei unterschiedliche Spezies identifiziert: das von Linné beschriebene Ullem als spezifische „Colica lapponica“ und die „Colica spasmodica Schleuchzeri“ als exemplarische Observatio und Typusbeleg der Spezies „Hepatalgia aeruginosa“ (1763, Bd. 4: 158).

Sauvages' Notizbuch diente also keineswegs nur als Blütensammlung oder Florilegium einer humanistischen Lese- und Wissenspraxis. Vergleicht man die Exzerpte mit den ursprünglichen Observationes sowie mit der publizierten Nosologie, so fällt auf, dass Sauvages hier wie an anderen Stellen sein Notizbuch nicht zitierte. Der gedruckte Eintrag des obigen Beispiels folgt fast wortwörtlich der ursprünglichen Textfassung der Linné'schen Flora lapponica, während alle Verkürzungen und Umstellungen des handschriftlichen Exzerpts scheinbar unberücksichtigt blieben. Bei der Überarbeitung der Nouvelles classes muss das Livre dennoch zur Hand gewesen sein, wenn auch nicht in der für die humanistische Naturforschung charakteristischen Weise in Form eines "storehouse of observations and experiments" (Yeo 2007: 11), sondern als methodus. Gleich einem Generator für neue Fragen trieb die sich in der Textarbeit manifestierende Differenz und Ähnlichkeit eine intensive Lektüre voran, die nachweisbare Spuren hinterließ. Die fragliche Beschreibung Scheuchzers suchte Sauvages im Original auf, denn in seinem Druckwerk wies 
er dessen Beschreibung - im Gegensatz zur zitierten Observatio Linnés - als seitengenaue Referenz nach. Mehr noch: Auch hier wurde bereits beim Exzerpieren die nächste Differenz markiert, wie das Fragezeichen zeigt: „Colica spasmodica Angelimontanorum Scheuchzeri, Iter Alpinum I. pag. 12. an Rachialgia metallica?" (1763, Bd. 4: 158).

Der Vorgang des Umtragens in ein zweigliedriges Bezeichnungssystem lässt sich mit dem Differenz erzeugenden Algorithmus einer symbolischen Maschine vergleichen, wie Sybille Krämer 1988 die inhärente prozedurale Verfahrenslogik von Formeln und Formalisierungen charakterisiert hat. Abbildung 3 zeigt die gewissermaßen blinde, weil freie Kombinatorik, mit der die binominale Benennung der gemeinsamen und unterscheidenden Merkmale (Gattung versus Art-Epitheton) eine Such- und Leseliste erzeugt. Hierfür gibt es im Livre zahlreiche Beispiele wie die listenförmigen Aufzählungen, die auf den ersten Blick den Schreibübungen eines Lateinschülers oder angehenden Medizinstudenten entsprungen zu sein scheinen. Auf einer halben Seite versammelte Sauvages fein säuberlich untereinander aufgereiht verschiedene Formen von Stummheit (Mutitas), die das Feld - von der Stummheit durch Würmerbefall über die von Galen beschriebene Stummheit nach Rekurrenslähmung bis hin zur Stummheit nach Apoplex - kartieren.

Noch länger ist die Liste für verschiedene Formen des Appetitlosigkeit (Anorexia). Sie umfasst elf Positionen möglicher Ursachen: von der Lähmung des Magens über die Melancholie bis hin zum Stupor. ${ }^{27}$ Bezüglich der Form ihrer Zusammenstellung gleichen diese Auflistungen dem Register einer

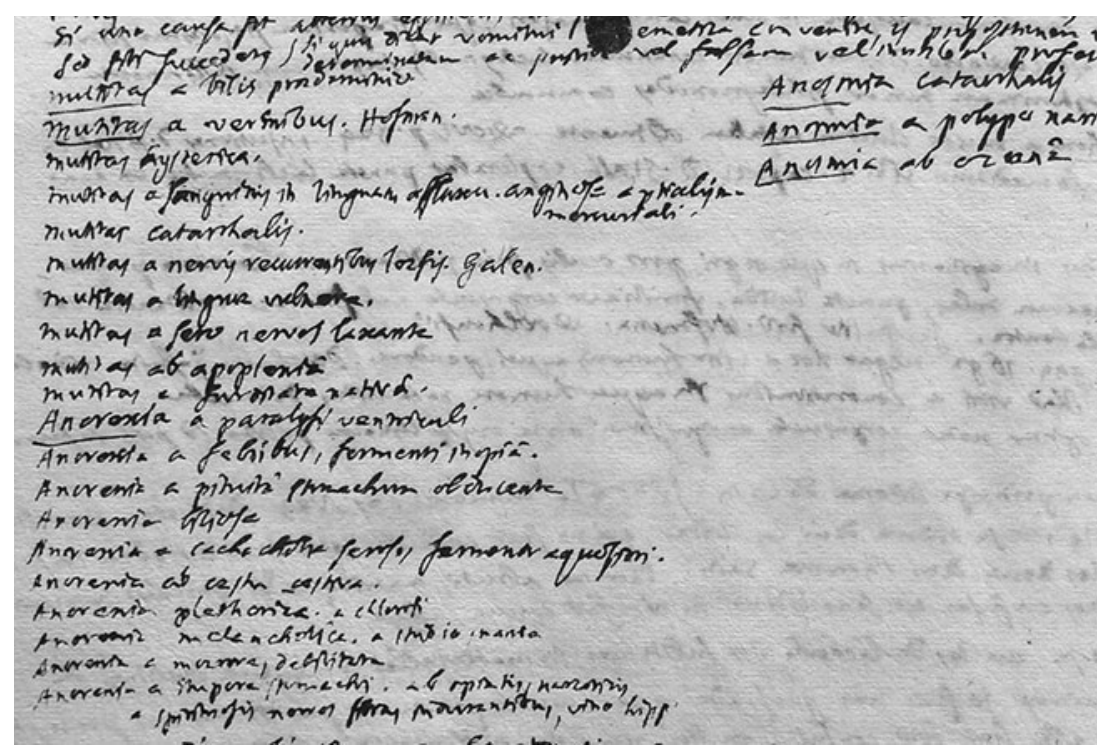

Abb. 3 Auschnitt aus der Liste zu verschiedenen Formen der Appetitlosigkeit ",Anorexia“ im Livre: 36 (Mit freundlicher Genehmigung des Archives départementales de l'Hérault). ${ }^{28}$ 
Tab. 1 Die Spezies der Anorexia in der Handschrift und im Druckwerk von Sauvages.

\begin{tabular}{|c|c|c|}
\hline Livre de raison (Winter 1747) & Pathologia (1759) & Nosologia (1763) \\
\hline Anorexia a paralysi ventriculi & Anorexia paralytica Sennert & $\begin{array}{l}\text { Anorexia } \\
\text { paralytica }\end{array}$ \\
\hline Anorexia a febribus, fermenti inopia & Anorexia febrilis Boerh. & Anorexia febrilis \\
\hline Anorexia a pituita phenacelum abducente & Anorexia a pituata Boerh. & Anorexia pituitosa \\
\hline Anorexia bilosa & Anorexia biliosa Bianchi & Anorexia biliosa \\
\hline $\begin{array}{l}\text { Anorexia a cachectiche serosa fermenta } \\
\text { aequistram. }\end{array}$ & & $\begin{array}{l}\text { Anorexia } \\
\text { cachectarum }\end{array}$ \\
\hline Anorexia ab cepha captiva & $\begin{array}{l}\text { Anorexia venere } \\
\text { exhaustorum }\end{array}$ & $\begin{array}{l}\text { Anorexia } \\
\text { exhaustorum }\end{array}$ \\
\hline Anorexia plethorica a clorosi & $\begin{array}{l}\text { Anorexia plethorica Bonet } \\
\text { sepulch. }\end{array}$ & $\begin{array}{l}\text { Anorexia } \\
\text { plethorica }\end{array}$ \\
\hline Anorecia melancholica, a studio mania & $\begin{array}{l}\text { Anorexia melancolica } \\
\text { Rammazin. }\end{array}$ & $\begin{array}{l}\text { Anorexia } \\
\text { melancolica }\end{array}$ \\
\hline Anorexia a mania, debilitate & Anorexia a saburra & $\begin{array}{l}\text { Anorexia a } \\
\text { saburra }\end{array}$ \\
\hline $\begin{array}{l}\text { Anorexia a stupore stomachi, ab operatis, } \\
\text { narcoticis }\end{array}$ & & Anorexia mirabiis \\
\hline \multirow[t]{3}{*}{$\begin{array}{l}\text { a spirituosis noxos fibras [unleserlich], } \\
\text { vide Lipp }\end{array}$} & & $\begin{array}{l}\text { Anorexia } \\
\quad \text { Neophytorum } \\
\text { Anorexia arthritica }\end{array}$ \\
\hline & Anorexia à pathematibus & \\
\hline & $\begin{array}{l}\text { Anorexia syphilitica D. } \\
\text { Cusson Obs. }\end{array}$ & \\
\hline
\end{tabular}

Sammlung von Observationes (Hess/Mendelsohn 2010: 288-291). Doch welchen Text indexieren diese Listen? Keinen, denn sie verweisen weder auf andere Eintragungen im Livre noch greifen sie dem Register der Pathologia (1759) oder Nosologia (1763) vor, wie Tab. 1 zeigt. Zwar werden einige der binominalen Bezeichnungen übernommen, doch belegen die auffälligen Unterschiede zwischen Manuskript und Druckschrift die mühsame Arbeit der kategorialen Präzisierung, die zwischen der im Winter 1747 handschriftlich festgehalten Liste und dem 1763 publizierten Register der Nosologia offenbar aufgebracht worden war (vgl. Tab. 1: Anorexia 1747-1763). Die Auflistung im Notizbuch organisierte sowohl die Arbeit in der Bibliothek, das Lesen und Exzerpieren unterschiedlicher Beobachtungen und Fallgeschichten, als auch die Arbeit an der Präzisierung der kategorialen Begriffe.

\section{Von der Fallgeschichte zur Historia}

Auch wenn Sauvages dem humanistischen Sammeln und Exzerpieren der Sammlungen von Observationes somit weitgehend treu blieb, so gab sein Methodus dem exzerpierten und zusammengetragenen Wissen trotzdem eine andere Form. Die Texttechnik des Exzerpierens vermochte, wie gezeigt, eine in 
der Literatur gefundene singuläre Observatio in einem mehrschrittigen Verfahren in mehrere Typusbelege verschiedener Spezies zu transformieren. Diese Form der Vervielfältigung der Kategorien ist ein bekannter Effekt solcher Differenzmaschinen. Die gleiche Technik tilgte aber auch empirische Vielfalt. Denn die Markierung der Differenzen und Gemeinsamkeiten war in der Lage, mehrere Observationes in eine singuläre Spezies zu überführen, wie wir nun zeigen wollen.

Anschaulich verfolgen lässt sich dies am Beispiel einer besonders klassischen Fieberart. Das bereits bei Hippokrates und Galen beschriebene Leipyria oder Eingeweide-Fieber (Juncker 1736: 156) hatte Sauvages - gleich einem Merkposten - schon in die erste Auflage der Nouvelles classes unter der Ordnung der remittierenden Fieber aufgelistet (1731: 66 „Triteophia leypiria. Manget. Bonet. Sepulchr."). Bildete dieser Topos 1731 noch eine - im wahrsten Sinne des Wortes - „Leerstelle“, die auf eine Observatio in Jean-Jacob Mangets (1652-1742) enzyklopädischer Sammlung verwies (1759: 260, Manget 1695: 444), so stieß Sauvages in den folgenden Jahren beim Sammeln und Exzerpieren offenbar auf eine aktuellere Fallsammlung, die ihm geeigneter schien, diese Lücke zu füllen.

Die in den Recueil d'Observations (1757) eingerückte Beschreibung von Johann Gottfried Hahn (1694-1753) ${ }^{29}$ folgte weitgehend der seit Thomas Sydenham (1624-1689) üblichen Darstellungsform einer Volkskrankheit. ${ }^{30}$ Eingehend ging der Dekan des Breslauer Medizinalkollegiums auf die lokalen Umstände ein, die dem Ausbruch der Volkskrankheit vorausgingen: den durchweichten Boden, die Überschwemmungen, die Wohnungsverhältnisse und Arbeitsbedingungen der armen Leute. Insbesondere aber gab der preußische Physikus einen gedrängten Überblick über das Wetter und die Witterung im Verlauf des Vorjahres, insbesondere auf die die Gesundheit gefährdenden nordwestlichen Winde. Erst vor diesem Panorama des epidemischen Genius loci präsentierte Hahn schließlich insgesamt elf Krankengeschichten, die er - je nach den ihm zur Verfügung stehenden Informationen - knapp bis ausführlich vorstellte, wobei er naturgemäß seine eigene Fallgeschichte in einer beeindruckenden Detailgenauigkeit erzählte. Jede dieser Observationes folgt dem Muster einer abgeschlossenen Fallgeschichte. Sie beginnt mit der Beschreibung der auffallenden Zeichen und Zufälle, resümiert die wesentlichen Veränderungen im Verlauf der Erkrankung und endet mit dem Ausgang in Genesung oder Tod: chronologisch organisierte Berichte, die geradewegs dem Krankenjournal des Physikus entsprungen zu sein scheinen. Tabelle 2 gibt eine Übersicht der ersten acht Observationes. ${ }^{31}$ Fall 1 war ein 50-jähriger Arbeiter, der an hohem Fieber, allgemeiner Erschöpfung, Kopf- und Herzschmerzen, galligem Durchfall und Schlaflosigkeit erkrankte, Fall 2 eine 30-jährige Jungfer, die ebenfalls an hohem Fieber, allgemeiner Bedrückung und schweren Kopfschmerzen litt, aber am zweiten Krankheitstag bei einsetzender Monatsblutung über galliges 


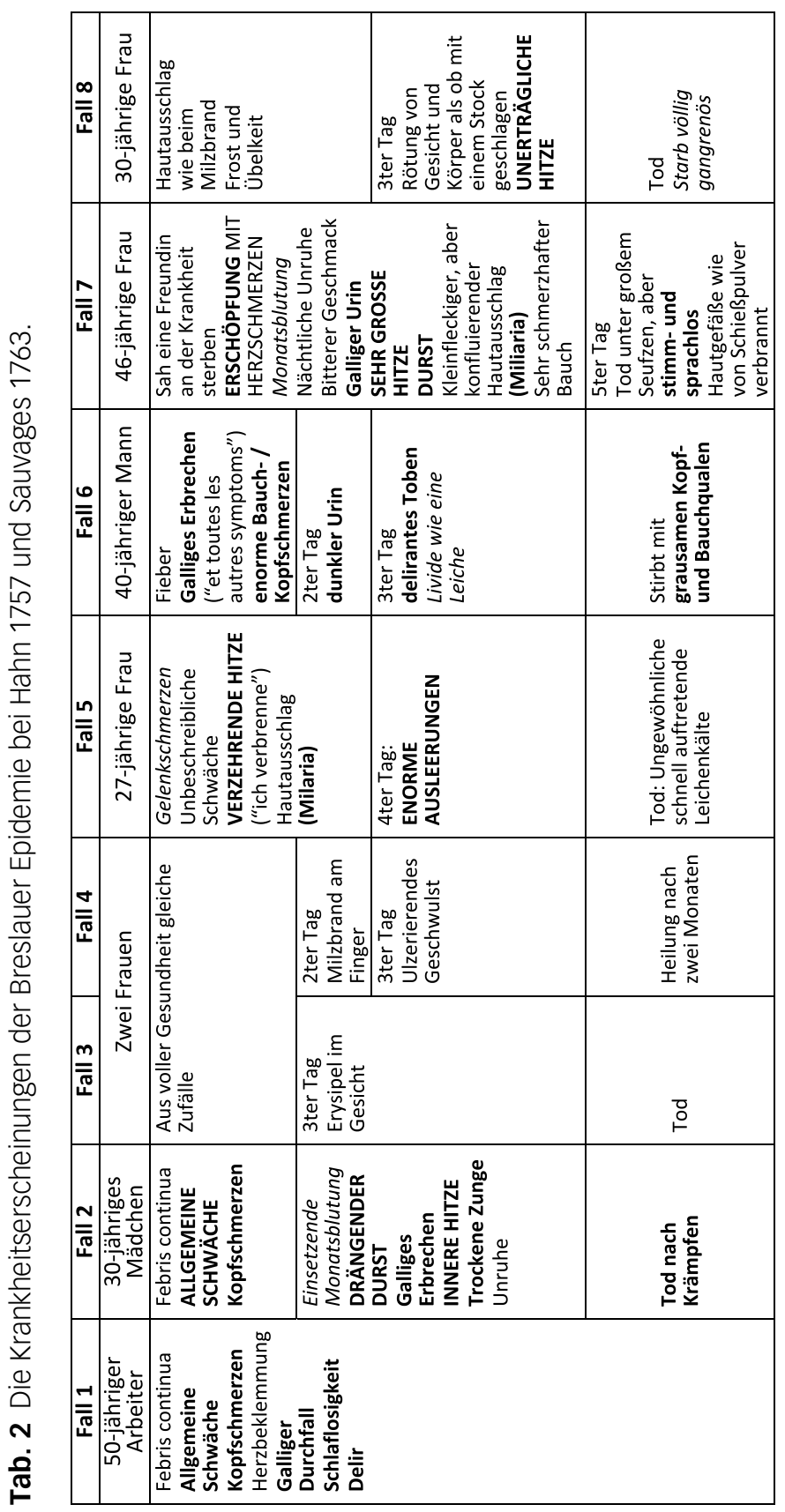


Erbrechen, aufdringlichen Durst, brennende innere Hitze und trockene Zunge klagte und schließlich unter Krämpfen starb. Fall 3 und 4 waren zwei aus voller Gesundheit an ähnlichen Zufällen erkrankte Frauen, von denen die eine bereits am dritten Tage einen Rotlauf im Gesicht zeigte und verstarb, die andere aber einen Milzbrand am Finger bekam und nach einem weitere Übel zwei Monate später vollständig genas. Dieser Reigen der meist tödlich verlaufenden Fallvignetten erhielt ihren dramatischen Höhepunkt durch Hahns eigene Krankengeschichte einschließlich glücklicher Heilung, die das Mitglied der Leopoldina über drei Seiten hinweg in peinlich genauer Selbstbeobachtung festhielt.

Von dieser Dramatik der narrativen Erzählung ist bei Sauvages nichts mehr zu spüren. Er exzerpierte die Fallgeschichten nicht als Observationes, sondern sichtete und prüfte nur die angeführten Zeichen und Zufälle. Ein knapper Paragraph referiert in der Vergangenheitsform die lokalen Umstände, dann folgt im präsentistischen Duktus eine subsumierende Zusammenfassung der zehn Observationes. Diese später auch als Précis beschriebene Exzerpiertechnik zielte nicht auf einen verkürzten Auszug der Observatio im Ganzen ab, sondern auf eine semiotische Differenzierung einzelner Merkmale. Den Algorithmus der Differenzmaschine haben wir versucht, synoptisch abzubilden (Tab. 2). Kursiv dargestellt sind jene Merkmale, die Sauvage beim Exzerpieren ignorierte und ausschloss, da sie nur in einzelnen Fallgeschichten auftauchen. In Großbuchstaben sind die unterscheidenden, in Fettschrift die als häufig beziehungsweise wiederkehrend ausgezogenen Merkmale zu erkennen, die Sauvages in einer Observation générale verdichtete. ${ }^{32}$ Am Ende hatte Sauvages' Methodus nicht nur Hahns 12-seitigen Bericht auf knappe zwei Seiten verkürzt, sondern zugleich in dreifacher Weise transformiert: Erstens hatte er die narrative Ordnung in eine serielle überführt, zweitens zehn singuläre Ereignisse oder Fallberichte in eine zeitlose Historia verwandelt sowie drittens dem namenlosen Grauen der Breslauer Epidemie, der mehr als 3.000 Menschen zum Opfer gefallen sein sollen, einen fest definierten Platz im medizinischen Kanon zugewiesen: „Diese Krankheit besteht aus einem akuten schweren fièvre lipyrie“ (1772, Bd. 2: 578). Selbst Hahns eigene Krankengeschichte wurde auf einen knapp einseitigen Eintrag verkürzt, in dem der preußische Arzt scheinbar selbst zu Wort kommt („Ipse Professor Hahn sequentia passus est"). Dabei brach Sauvages die ausführliche Selbstbeobachtung jedoch auf folgende tabellenförmige Aufzählung um:

1. die accessus febrilis vehemens, [...]

Die 3. dolores sedebantur; nox pessima.

Die 4. omnia pejus habuere [...]

48. desquamatio cutis, unguium lapsus. (1763, Bd. 2: 295 f.)

Durch diese dreifache Transformation wurde aus der Beschreibung einer unbekannten Seuche in Breslau, der „déscription d'une épidémie“ (Hahn 1757: 452), die systematische Darstellung einer Tritaeophia vratislaviensis. Das 
Exzerpt der elf Observationes erhielt somit - gleich der Colica lapponica - den Status einer eigenen Spezies, die sich durch eine charakteristische Chronologie der Kombination von Fieber und allgemeiner Schwäche von anderen Fieberarten (siehe Tab. 2, in Großbuchstaben) unterschied. Beide, die gemeinsamen und unterscheidenden, Merkmale reduzieren Hahns Fallsammlung schließlich auf eine präzise, knapp 17-zeilige Definition: „eine allgemeine Schwäche, Schmerz im Kopf und im Herzbereich, seröser galliger Durchfall, Schlaflosigkeit, ein Irresein das toll ist und von heftiger Angst begleitet wird; am folgenden Tag ungestümer Durst, Erbrechen und galliger Durchfall ... [schließlich tritt] epileptischer Tod [ein]“ (Sauvages 1763, Bd. 2: 295). ${ }^{33}$

Nichts könnte den Unterschied zur bisherigen Praxis des Exzerpierens und Sammelns drastischer illustrieren als diese Form der Verallgemeinerung. Wo Pieter van Foreest, Fabricius Hildanus oder Théophile Bonet sich die Gelegenheit nicht hätten entgehen lassen, der Hahn'schen Beobachtung einer Epidemie exzerpierend mehrere Fallgeschichten $\mathrm{zu}$ entnehmen und der eigenen Observationes-Sammlungen an der einen oder anderen Stelle einzufügen, ${ }^{34}$ da verdichtete Sauvages' Exzerpt die singulären Fallgeschichten zu einer Histoire générale. Er verwendete diesen Begriff explizit zwar nur für die Definition der Klasse (1772, Bd. 2: 274). Doch es war die gleiche Schreibtechnik, die auch die partikularen Observationes in eine verallgemeinernde Form überführte. Der Auszug erfasst die Geschichte einer Krankheit nicht mehr in ihrem Ereignischarakter. Das Exzerpt begriff die Fülle der Historia auch nicht länger als Ansammlung vergleichbarer Fälle unter einem zugehörigen Topos. Stattdessen eliminierte ein Zweischritt just jenen exemplarischen Charakter einer Beschreibung, die keinen Unterschied zwischen Historia und Observatio kennt. ${ }^{35}$ Vergleich, Isolierung, Markierung und Zusammenstellung der eine Spezies beschreibenden Symptomata erzeugten eine verallgemeinernde Darstellung. Damit verlor die Historia ihre synonyme Bedeutung zur Observatio, die ihr im Humanismus noch zugekommen war (Pomata 2005). Diese Form eines generalisierenden Exzerpierens bildet eine spezifische paper technology aus, die wenige Jahrzehnte später im Schreibbüro der Société Royale de Médecine und von den korrespondierenden Mitgliedern in der Provinz praktiziert werden sollte (Mendelsohn 2011). Diese Art der Historia enthebt den konkreten Fall seiner singulären Evidenz, ordnet seine Merkmale in einer Reihe an und fügt ihn damit in eine allgemeine Darstellung ein (Hess/Mendelsohn 2010).

\section{Von der Historia zur Klassifikation}

Wie vor ihm die humanistischen Ärzte, so sichtete auch Sauvages die medizinische Literatur und durchkämmte alle ihm greifbaren Fallsammlungen. 
Dabei stützte er sich, wie das Livre zeigt, bevorzugt auf neuere Publikationen. Mit der chronologischen Abfolge seiner Aufzeichnungen dokumentiert Sauvages' Notizbuch somit die Bewegungen im Lesen wie im Denken. Um diesen Prozess zu rekonstruieren haben wir die Eintragungen - Exzerpte, Krankengeschichten, aber auch theoretische Reflexionen wie beispielsweise über den ontologischen Status der Krankheitsart - den gedruckten Ausführungen zugeordnet.

Die Nouvelles classes dienten, um ein erstes Zwischenresümee zu ziehen, nicht als Loci communes. Dagegen spricht die Unordnung des Livre. Die Classes stellten aber ein förmliches Raster verallgemeinernder Begriffe bereit: Sauvages' binominale Nomenklatur definierte mit der von Tourneforts' entlehnten Gattungsbezeichnung (Martin 1990: 120) die gemeinsamen Merkmale, während das Epitheton die unterscheidenden Kriterien festlegte. ${ }^{36}$ So basierte die Definition des regelhaft jeden zweiten Tag auftretenden einfachen Fiebers (Quotidiana simplex) auf der klassischen Beobachtung Sydenhams, von der alle weiteren unterschieden wurden. Das schleichend einsetzende Quotidianfieber aus den Curationes Wolfgang Gabelkovers (Febris intrans), das gedoppelte Quotidianfieber, eine von Daniel Sennert (1572-1637) beschriebene seltene Verlaufsform (Sennert 1676: 437) oder die Beobachtung einer noch selteneren dreifachen Form. Diese hatte Sauvages in der Fieberabhandlung von James Primrose (gest. 1659) aufgespürt (Primrose 1658) und in seine Liste übernommen, jedoch ihren systematischen Stellenwert bezweifelt und sie als „erdacht“ bezeichnet („est imaginée“ (1731: 38)). Auf diese Weise war es Sauvages gelungen, insgesamt 13 Fieberarten der Gattung Quotidiana zu unterscheiden und zu definieren (ebd.: 36-39). Man könnte meinen, das begriffliche Raster wäre damit entwickelt. Doch weit gefehlt: Die Arbeit am System hatte damit erst begonnen!

Die Tab. 3 gibt nur einen schwachen Eindruck von den Anstrengungen, die Sauvages in den folgenden Ausgaben unternahm, um das in den Nouvelles classes kompilierte Wissen in mehrfachen Schleifen zu verarbeiten, zu tilgen, neu $\mathrm{zu}$ formulieren und schließlich zusammenzustellen. Der Transformationsprozess klassischer Beschreibungen à la Sennert, Primrose und Gabelkover in systematische Kategorien (duplex, deceptiva) ist manchmal nur mühsam zu verfolgen, wie das weiter unten dargestellte Beispiel der Febris subcontinua malignans zeigt. Während die beiden folgenden Auflagen von 1739 (S. 112) und 1752 (S. 213) keine Angaben zu den Spezies einer Gattung gaben, listete die Pathologia methodica, wenn auch in tabellarischer Form, wieder die einzelnen Spezies auf (1759: 261 f.). In diesen späteren Auflagen zeigt sich sehr schön die systematisierende Funktion. Mit jeder Neuauflage wurden zwei, drei oder mehr Spezies eliminiert. So fehlen 1759 beispielsweise die Formen der Complicata von 1731, weil sie, wie Sauvage in der späteren Ausgabe (1763, Bd. 2: 314) lapidar anmerkt, in der Quotidiana duplex enthalten seien. 


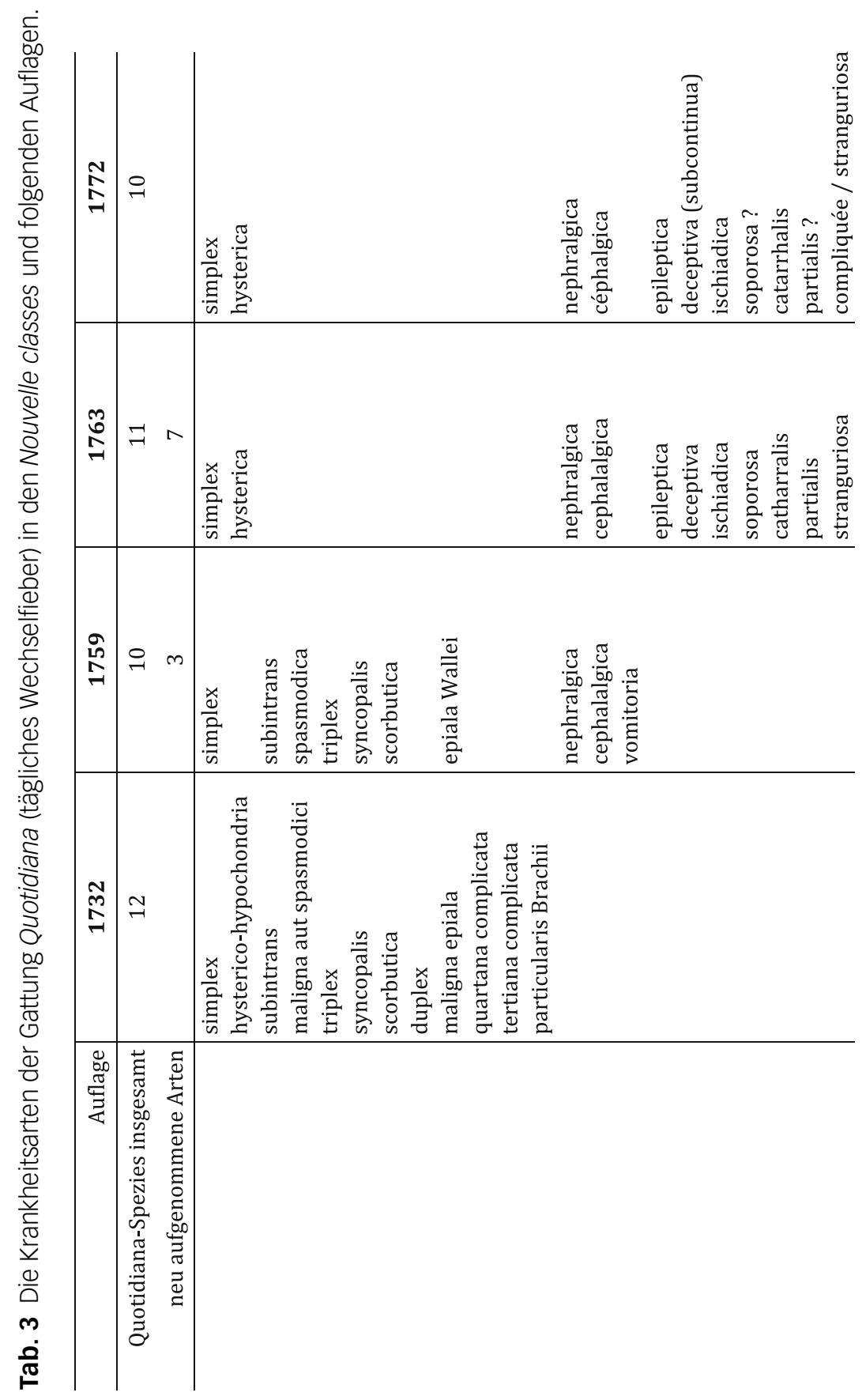


Die Differenzmaschine der methodischen Textarbeit verdichtete auf diese Weise die Krankheitsarten. Eine Schritt für Schritt allgemeinere Definition zog Auflage um Auflage immer mehr Observationes zusammen und subsumierte sie unter einer einheitlichen Bezeichnung. Die Textarbeit blieb jedoch Stückwerk, wie die Tabelle zeigt. Auch Sauvages traf der Fluch des humanistischen Lesens und Sammelns (Blair 2010b). In jeder Auflage hatte er mit mehr Informationen zu kämpfen. Er führte neue Observationes auf, die beim Exzerpieren unter die Gattung der Quotidiana eingeordnet worden waren. In der Nosologia kamen die Quotidiana soporosa, catarrhalis, partialis und stranguriosa (1763, Bd. 2: 317 f.) hinzu, von denen in der letzten Auflage eine Spezies wieder getilgt und die anderen mit Fragezeichen versehen, also für die weitere Textkritik vorgemerkt wurden (1772, Bd. 2: 620 f.). Unablässig wälzte Sauvages die medizinische Literatur. Gleich Sisyphos gelang ihm der Abschluss seiner Arbeit jedoch nie.

Im Unterschied zu anderen Systematikern scheiterte Sauvages nicht an der Fülle. ${ }^{37}$ Während Carl Linné mit jeder Ausgabe der Systema naturae die Zahl der Gattungen und Arten weiter steigerte, ${ }^{38}$ verzeichnete auch die Nosologie méthodique letzter Hand nicht wesentlich mehr Spezies als zu Beginn des Projekts im Jahre 1731. Natürlich wuchsen die Nouvelles classes von der ersten Auflage an beständig und fast exponentiell an und entfalteten sich zu einem zuletzt Regal füllenden zehnbändigen Werk mit jeweils über 500-seitigen Bänden. Der allseits beklagte Informationsüberfluss galt jedoch nur für die Beschreibung der einzelnen Spezies, die von knappen ein- bis dreizeiligen Einträgen zu langen, fast schon monografischen Abhandlungen expandierten. In diese Historia gingen all die Informationen zum Verlauf der Erkrankung, Disposition und Komplikationen, Behandlung und mögliche Folgeerkrankungen ein, die Sauvages im Laufe der Jahre gesammelt hatte. ${ }^{39}$

Sauvages verzichtete somit nicht auf die Papiertechniken der humanistischen Textarbeit. Diese entfalteten jedoch andere Effekte. So erhielt das Sammeln medizinischer Fallgeschichten eine neue epistemische Bedeutung. Nicht die Menge der Beobachtungen, das Anhäufen der nächsten hundert Observationes zum Druck, sondern die Ordnung der in ihnen enthaltenen Erfahrungen war das Ziel des extensiven Literaturstudiums. Und Ordnen bedeutete ganz konkret eben nicht, eine Observatio - gleich einem seltenen Käfer oder kostbaren Mineral - in das passende Schächtelchen eines ExzerptKastens (Meinel 1995) oder Naturalienschranks (te Heesen 2000) zu sortieren, sondern das passende Schächtelchen durch den Vergleich der Merkmale im oben beschriebenen Zweischritt erst herzustellen. Dabei standen - wenn man in diesem Bild bleibt - das Sammlungsobjekt und seine Aufbewahrungsbox in einem beständigen Wechsel. Fand sich für eine neue Observatio eine passende Box, gab es also das Schächtelchen für diesen Käfer bereits, dann verlor der Käfer seinen Reiz als Sammelobjekt. Eine Fallgeschichte wurde höchstens sofern sie zu den bedeutenderen Sammelobjekten gehörte - als weitere 
Belegstelle referiert. ${ }^{40}$ So ging das bereits erwähnte „schleichend einsetzende Quotidian-Fieber", das zu den ersten eliminierten Fieberarten zählte, im „täuschenden“ eintägigen Wechselfieber (Quotidiana deceptiva) auf, das Sauvages sowohl auf einer Fallsammlung von Francesco Torti (1658-1741) als auch auf der „Febris intrans Auctorum“ (verschiedener Autoren) begründete - und damit Gabelkovers Fallgeschichte subsumierte (siehe Tab. 3).

Diese 1763 neu eingeführte Fieberart schloss aber nicht nur zwei traditionelle Fieberbezeichnungen ein, sondern bildete gewissermaßen in sich eine Differenzkategorie. Obwohl diese Fieberart einem wahren eintägigen Wechselfieber entspräche, seien „l'intermission \& le type [...] très obscurs“ (Sauvages 1772, Bd. 2: 617) und hätten sich in der Abgrenzung zum gedoppelten TertianFieber der bösartigen Art (Febris subcontinua malignans) zu erweisen. ${ }^{41}$

Die Nosologie war somit nicht das Produkt einer abgeschlossenen Arbeit, die in mühsamer Schreibarbeit hinter den Kulissen in Notizbüchern, Krankenjournalen oder Loci communes verrichtet wurde. Vielmehr waren die Veröffentlichungen selbst ein Arbeitsinstrument. Im Unterschied zur Johns Kritik an der vorgeblichen Vereindeutigung und Verfestigung des Wissens durch die Einführung des Buchdrucks (Johns 1998, Baron/Lindquist/Shevlin 2007) wollen wir auf die Bedeutung der überarbeiteten Auflagen im Erkenntnisprozess hinweisen. Während Linné im persönlichen Handexemplar seiner Systema durch die bereits im frühen 18. Jahrhundert verbreitete Technik (Blair 2004: 96) eingeschossener Leerseiten jenen Platz für handschriftliche Annotationen gewann, mit dem er den enormen Zuwachs seiner Klassifikation organisierte (Müller-Wille/Scharf 2011), war es bei Sauvages die Druckfassung selbst, die den jeweils erreichten Zwischenstand als Arbeitsinstrument eines zirkulären Überprüfens, Verwerfens und Hinzufügens festhielt. Die Nouvelles classes stellten kein vorläufiges Ergebnis dar, sondern bei jeder erweiterten Auflage erneut den für einen Moment geronnenen Prozess in einer weiteren Schleife der kritischen Evaluation.

Diese drei Elemente - Reduktion der Fülle durch Generalisierung, ${ }^{42}$ Indexierung als kategoriale Arbeit an der Wissensordnung und der Einsatz des Drucks als Verfahren der Wissensproduktion - unterscheiden Sauvages' paper technology von den humanistischen Textmethoden. Mit Blick auf den Aufwand, den beispielsweise Ulisse Aldrovandi (1522-1605) betrieb, um die zunächst in Säcke gestopften Exzerptzettel in eine Ordnung zu überführen, imponiert die Flexibilität von Sauvages' Aufschreibesystem aus handschriftlichem wie gedrucktem Papier. Während es dem Humanisten Aldrovandi trotz mehrerer Dutzend dicker Folianten aus gutem Papier und dem richtigen Kleber (Krämer 2013) nicht gelang, sein Vorhaben in eine gedruckte Ausgabe zu überführen, präsentierte Sauvages' hybrides, aus verschiedenen Texttechniken zusammengefügtes Aufschreibesystem mit jeder neuen Auflage der Nouvelles classes erneut ein Wissen, das sich - befreit von einer mühsam mit 
Kleber fixierten oder gar in Blei gegossenen Ordnung - dem raschen Wandel der medizinischen Erfahrung als gewachsen erweisen sollte.

\section{Vom Faktoid zur Krankheitsspezies}

Mit diesem Rüstzeug können wir uns nun noch einmal der Frage zuwenden, der wir oben ausgewichen sind: Welche Auswirkungen auf das Verständnis der Krankheit zog die Anwendung einer Methode „semblable à celui des Botanistes" nach sich? Die geläufige Antwort scheint einfach (Hess 1993: 1518): Mit der Durchsetzung der nosologischen Klassifikation begannen die Ärzte das Reich des Krankseins gleich der lebenden Natur in abgrenzbare Entitäten einzuteilen. Bis heute ruht kein Forscher, bevor nicht jede Erkrankung ihren Platz in der medizinischen Wissensordnung gefunden hat und es gibt keine Krankheit, die nicht gleich einer Spezies aus Flora oder Fauna systematisch klassifiziert werden könnte. Doch Sauvages' methodisches Vorgehen begründet keinen ontologischen Krankheitsbegriff. Damit ist die Frage über den ontologischen Status des Krankseins zwar nicht beantwortet. Doch der Transformation der Observatio in die Spezies einer systematischen Klassifikation kommt in der Entwicklung des medizinischen Wissens eine andere Bedeutung zu als der Ontologisierung der Krankheit, da sie dem Faktischen der Beobachtung - hier sei an die Interpretation der Observatio als Faktoid erinnert (Blair 1992: 545 f.) - einen neuen empirischen Status verlieh.

\section{Die Entstehung der Klassifikation aus humanistischer Schreibarbeit}

Verallgemeinerung statt Sammeln, Begriffsarbeit statt Fülle und Vorläufigkeit statt Permanenz: Sauvages' Aufschreibesystem steht für die Ablösung der humanistischen Historia durch ein nosologisches Klassifikationssystem. Wie gerade dieses prominente Beispiel zeigt, war der historische Prozess weniger von der Botanik beeinflusst (oder gar bedingt) als mit der Entwicklung einer paper technology verwoben. So stehen Sauvages Aufschriebe weit mehr in der Tradition des humanistischen Unternehmens als dies bisher in der Forschungsliteratur wahrgenommen wurde. Die zehnbändige Nosologie stellt sich aus unserer Sicht - etwas zugespitzt - weniger als das Resultat des Niedergangs und Verfalls der humanistischen Schreib- und Bibliotheksarbeit als vielmehr deren Krönung dar: eine in jahrzehntelanger Arbeit des Lesens und Exzerpierens, Sammelns und Systematisierens zusammengetragene Ordnung des medizinischen Wissens. Aber nicht nur Sauvages' Anspruch steht in dieser humanistischen Tradition. Auch die von ihm eingesetzten Praktiken sind (wie die Vorgeschichte des Livre zeigt) aus der humanistischen paper technology erwachsen. Die im 18. Jahrhundert aufblühende Klassifikation ist - ob nun in der Medizin oder in der Naturgeschichte - kein gänzlich neues Projekt. Trotz 
der neuen Wissenschaften, trotz der Revolution des Wissens in Botanik, Physiologie, Chemie und Physik vollzieht die Klassifikation nur in Teilen einen Bruch mit der humanistischen Methodik. Besser: Die naturhistorische Klassifikation repräsentiert eine Reform der humanistischen Textarbeit, mit der sich die Frage nach dem Ende des Humanismus neu stellt.

Kein Bruch, so lautet unser historisches Argument, sondern die Entfaltung und Weiterentwicklung des Gebrauchs von Papier, Feder und Tinte beim Lesen, Exzerpieren, Ordnen und Vervielfältigen: Das spezifisch Humanistische dieser Schreibpraktiken lässt sich mit Blair 2010a, Yeo 2010 und Stolberg 2013 etwas verkürzend mit einer besonderen Form des note-taking (te Heesen 2005b) charakterisieren: Wissenschaftlich „Notiz nehmen“ und „Notizen machen" erfolgte in der Renaissance als Anfertigung von Loci communes, ob nun im systematischen oder sequentiellen Typus (Stolberg 2013). Wenn man die Technik der Loci communes aber als gemeinsame materiale Grundlage des humanistischen Wissens begreift, dann verweist die eingangs erwähnte Charakterisierung von Sauvages' Notizbuch als Potpourri auf die offenbar entscheidende Differenz, die das Sammeln und Ordnen in einem Zeitalter der naturhistorischen Klassifikationen von der humanistischen Wissensform der Historia trennen sollte. Mehr noch: Berücksichtigt man, dass das Livre die zweite oder gar dritte Etappe der Verschriftlichung von Beobachtungen seien es eigene oder angelesene - bildete, dann wiegt Sauvages' Verzicht auf die vertrauten Loci communes umso schwerer. Das Livre zählt aber auch nicht zu den Adversaria, in denen Erinnerungswertes ungeordnet festgehalten wurde (ebd.). Vielmehr vermerkte Sauvages seine Beobachtungen am Krankenbett oder Sektionstisch mit Sicherheit gesondert, bevor er sie in das uns überlieferte Notizbuch übertrug. ${ }^{43}$ Dabei schrieb er sie in der zeitlichen Folge ihrer Verfertigung ein. Die Chronologie erzeugte hier auch eine Ordnung. Sie sortierte zwar nicht das „Machen der Notizen“, hielt aber die Ordnung des „Notiz nehmens" fest. Das Livre gleicht somit einem Merk- oder Gedankenbuch, das in der chronologischen Aufzeichnung die Verfertigung und Verfestigung von Beobachtungen, Überlegungen und Erkenntnissen dokumentiert (Hoffmann 2010). In diesem Sinne stellen Papiertechniken eine Arbeit am System dar, die in eben dieser Prozesshaftigkeit selbst eine ordnende Struktur herausbilden.

\section{Die Ordnung des Beschriebenen}

Die Frage nach dem ontologischen Status der auf diese Weise verfertigten und verfestigten Wissensobjekte wird durch Verlagerung der Perspektive (von der Ordnung des Sammelns hin zu einer Ordnung des Schreibens) provoziert. Daher sollte sie auch mit Blick auf die Praxis des Sammelns, Ordnens und Klassifizierens beantwortet werden, nämlich, um das oben bemühte Bild noch einmal zu strapazieren, als Frage nach dem Status von Käfer und Schachtel, wenn der Käfer im Zuge des Sortierens zum Schächtelchen mutiert. Diese Frage ist aus zwei Gründen nicht trivial. Zum einen gilt die Unterscheidung 
der Arten, Gattungen, Ordnungen und Klassen von Krankheiten als Ausweis eines ontologischen oder substantiellen Krankheitsbegriffs (Wieland 1975, Williams 2003), womit der Inhalt dieser klassifikatorischen Bemühungen - die Krankheit - zumindest tendenziell mit den besagten Käfern oder Mineralien gleichgesetzt wird. ${ }^{44}$ Zum anderen wird dem Genre der Darstellung von Krankheiten eine entscheidende Bedeutung bei der Renaissance des empirischen Wissens im 17. Jahrhundert zugemessen, bei dem die neuere Forschung die Observatio als neues epistemisches Genre bestimmt hat (Daston/Lunbeck 2011). Welchen Status hat nun der Gegenstand der Nosologie?

Wenden wir uns also ein letztes Mal den Gegenständen von Sauvages' Schreibarbeit zu - und zwar dem systematischen Doppelgänger des bereits ausführlich diskutierten leipyrischen Fiebers. Als Hahns „déscription d'une épidémie“ 1763 einen Namen und Platz in den Nouvelles classes erhielt, eliminierte Sauvages nicht nur diese Leerstelle, sondern zugleich auch deren nach der Logik der nosologischen Benennung zwingend folgende Parallelform. Denn Sauvage hatte für jenes Fieber, das „zur Entzündung derer innern Theile schläget", 45 zwei Loci vorgehalten: Wenn das Fieber im täglichen Verlauf stark schwankte (remittens), wurde die zugehörige Observatio den remittierenden Fiebern zugeordnet, wenn es ganz aussetzte (intermittens), der Gattung der Wechselfieber. Für die Febres remittentes zog Sauvages (1759: 260) eine Fallgeschichte von Jean Manget heran (die dieser zu den Febres continuae lethales zählte (Manget 1698: 443)). Dieser Zuordnung widersprach aber der Beobachtung in den von Jacques Thévart (1600-1674?) herausgegebenen Consilia, in der sich die Leipyria mit einem galligen Brechdurchfall (Cholera) abwechselte (Baillou 1640: Consilium LV, 228-231). Diesen Widerspruch hatte Sauvages pragmatisch mit dem Eintrag einer zweiten Leipyria in der Gattung der Wechselfieber (1759: 262) aufgelöst. Nicht die Observatio, die sich der Ordnung sperrt, wurde wie einst bei Bonet abgeschrieben und verdoppelt (Hess/Mendelsohn 2010: 289). Stattdessen wurde das Schächtelchen dupliziert und ein zweiter Topos oder locus im Sammlungsschrank markiert. Hier behandelte Sauvages die in der Literatur gefundenen Observationes gleich einem Käfer wie ein Objekt der Naturgeschichte.

Aber Sammelobjekt und Aufbewahrungsschachtel konstituierten sich wechselseitig. Das durch die bis dahin unbekannte leipyrische Fieberepidemie definierte und neu eingeführte Schächtelchen (Tritaeophia vratislaviensis) zog im methodischen Zweischritt unweigerlich die Neudefinition der anderen Schächtelchen dieser Fiebergattung nach sich. Dies hatte offenbar zur Folge, dass beide Käfer - die Leipyria remittens von Manget und die Leipyria intermittens von Thévart - aus der Ordnung fielen, allerdings mit unterschiedlichem Schicksal. Für Mangets Schächtelchen fand Sauvages eine besser passende Observatio, für das Schächtelchen von Thévarts Consilium hingegen nicht, weshalb es diese Schleife der Revision nicht überstand. Das Verhältnis von Kategorie und Krankheit kehrte sich folglich um. Nun war es 
das Schächtelchen, das die Sammelobjekte definierte. Die im Zuge dieser Begriffsarbeit geschärfte Tritaeophia leipyria wurde 1763 mit Beobachtungen und Fallberichten von Galen, Petrus Gorraeus, Aetius und Pieter van Foreest gefüllt und stellte damit ihre praktische Berechtigung - zum Teil mit seitengenauer Referenzangabe - unter Beweis.

\section{Krankheitsspezies aus Papier}

Die Umkehrung - von einem der Ordnung vorausgehenden in ein durch die Ordnung definiertes und substantiiertes Objekt - vollzog sich nicht nur im übertragenen Sinne auf dem Papier. Sie war vielmehr - wie die lange Autorenliste von Avicenna bis Valcaringhi veranschaulicht - das Ergebnis eines intensiven Lesens, Exzerpierens und Umschreibens. Folgt man jedoch Sauvages' Verweisen, dann reduziert sich die angegebene Literatur rasch auf einen Gewährsmann, nämlich Pieter van Foreest (1521-1597). Der als holländischer Hippokrates gerühmte Delfter Stadtphysikus hatte Ende des 16. Jahrhunderts eine riesige Sammlung von Observationes zusammengetragen, in der sich auch die Fallgeschichte eines tödlichen leipyrischen Fiebers findet. Sie enthält die meisten Autoren und Referenzen, die auch Sauvages anführt (Foreest 1584: 291-299, obs. 36).

Foreests Observatio ist aber nicht nur wegen dieses Einblicks in Sauvages Arbeitstechnik spannend. Seine Fallsammlung gilt als Meilenstein in der Entwicklung der medizinischen Fallgeschichten $\mathrm{zu}$ einem epistemischen Genre (Pomata 2011), der sich bereits im formalen Aufbau der Observatio manifestiert. Zuerst erzählte er in beeindruckender Ausführlichkeit und chronologischer Abfolge die Krankengeschichte einer 26-jährigen Delfter Dienstmagd. Dieser über fünf Seiten langen Krankengeschichte folgte dann, abgesetzt durch eine Überschrift, ein dreiseitiges Scholion, in dem die überlieferten Lehrmeinungen vorgestellt, verglichen und im Bezug auf die eigenen Beobachtungen diskutiert werden.

Diese Anordnung von Observatio und Scholion war neu. Sie charakterisiert den Empirismus der Renaissancemedizin. Bis dahin ging der empirischen Beobachtung die theoretische Erörterung voraus. Erst Ärzten wie Amato Lusitano (1511-1568) war es gelungen, diese Ordnung von Gelehrsamkeit und Krankengeschichte, von Theorie und Praxis, von überliefertem Wissen und Erfahrungswissen gewissermaßen vom Kopf auf die Füße zu stellen. Sie zogen die am Krankenbett aufgezeichnete Fallgeschichte dem Scholion vor, so dass die gelehrte Erörterung der überlieferten Autoritäten als erläuternder Kommentar am Ende der Observatio folgte (Pomata 2010). Diese Umkehrung zog aber nicht nur symbolisch die Blickwendung vom Buch zum Bett nach sich. Sie schloss auch eine Abkehr von der geläufigen Ordnung dieses Buchwissens ein, die mit dem Bedeutungszuwachs der Fallgeschichte einherging. Die Observatio stellte hierbei sowohl das narrative Format als auch die grundlegende Einheit und Währung dieses neuen Wissens bereit. Denn sie transportierte 
nicht nur die empirischen Fakten. Sie repräsentierte diese vielmehr - als Observatio - selbst (Daston 2011).

Doch was machte Sauvages mit dieser Neuerung? Wie verhielt er sich zu diesem Empirismus des Humanismus? Er stellte ihn erneut auf den Kopf. Auf ganze neun Zeilen verkürzte Sauvages Foreests seitenlange Observatio und reduzierte sie auf eine allgemeine Definition: „Dies ist eine Abart der Tritaephia causonis seu ardentis, bei dem die Extremitäten kalt sind, während die Hitze den ganzen Leib einnimmt" (Sauvages 1763, Bd. 2: 300). Nur im Verweis („wie im Fall von Foreest") war die Definition noch als Auszug einer konkreten Krankengeschichte zu erkennen. Auch die darauf folgende Abhandlung erinnert eher an eine gelehrte Auseinandersetzung: Hier erörtert Sauvages ob die Lehrmeinungen der auctoritas - von Avicenna über Baglivi bis Valcaringhi - dieser Definition der Leipyria gerecht werden. Erst im letzten Absatz referiert er schließlich eigene Beobachtungen mit dieser schwierigen Fieberart (ebd.: 301).

Hatten Amato und die anderen Gelehrten der Renaissance das überlieferte Wissen gewissermaßen an den Rand der Observatio gedrängt, so kehrte Sauvages diese Beziehung zwischen allgemeiner Darstellung und kasuistischer Beobachtung erneut um und definierte zugleich das "gelehrte Wissen" als eine Generalisierung der empirischen Beobachtung. In den Nouvelles classes lieferte Sauvages eigene Beobachtung in Form einer Randbemerkung den Anlass für eine längere Überlegung über den epistemischen Status dieser species: All das, was die vorliegende Literatur biete, so kritisierte Sauvages, seien nur singuläre Krankengeschichten und stellten wohl Varietäten, aber keine eigene Spezies dar. Die Methode stehe damit aber nicht in Frage. Viele individuelle Erkrankungen dieser Gattung seien sich in Verlauf, Typus, Ausgang und beständigen Symptomen gleich, weshalb man sie zum Gewinn der Medizin als Spezies beschreibe (describi).

Die Frage nach dem ontologischen Status der Krankheit ist somit, wie wir zeigen wollten, nicht historisch zu beantworten. Für Sauvages und seine Sammelobjekte stellt sie sich nicht, obwohl er wie seine Zeitgenossen die am Bett beobachteten Krankheitsverläufe nach Arten, Gattungen und Ordnungen einzuteilen begann. Das historisch entscheidende Moment bildete hierbei jedoch der Prozess des Verfertigens, in dem sich die kategoriale Definition und das Objekt des Sammelns wechselseitig bestimmten. Etwas pointiert lässt sich folglich sagen, dass die Spezies einer Krankheit eine Kunst des Schreibens ist, nämlich das Produkt einer techne - und die ontologische oder substantielle Krankheitsentität letztlich eine Frage der dabei eingesetzten Techniken des Papiers. 


\section{Danksagung}

Der vorliegende Beitrag entstand im Rahmen des vom European Research Council geförderten Forschungsvorhabens „Ways of Writing. How Physicians Know“. Erste Fassungen wurden auf der Jahrestagung der Deutschen Gesellschaft für Geschichte der Medizin, Naturwissenschaften und Technik in Stuttgart 2011 sowie der Konferenz „Worlds of Paper“ in London 2012 zur Diskussion gestellt. Der Dank für die Hilfe bei den Übersetzungen gilt ConradJakob Schiffner, für Anregungen und Kommentare Ruth Schilling, Michael Stolberg, Staffan Müller-Wille sowie den unbekannten Gutachtern und Gutachterinnen der NTM.

\section{Anmerkungen}

1 Archives départementales de l'Hérault (im Folgenden: ADH), $10 \mathrm{~F}$ 51. Williams geht in ihrer Arbeit über den Vitalismus in Montpellier kurz auf das Livre ein (2003: 33, 95).

2 ADH, $10 \mathrm{~F} 53$.

3 Von der sehr raren ersten Auflage sind zwei Fassungen aus Avignon (1731, 1732) nachgewiesen. Im Folgenden zitieren wir die Auflage von 1731 der Universitätsbibliothek Erlangen.

4 Siehe hierzu te Heesen 2005a, Müller-Wille/Scharf 2011 und die Beiträge im gleichen Heft (NTM N. S., 21 (2013), Heft 1: Paper Technology in der Frühen Neuzeit).

5 Das Hausbuch (Livre de Raison) wird zumindest in späteren Zeiten mit dem Aufschreibeformat der Kladde, des Sudel- oder Gedankenbuchs (Georg Christoph Lichtenberg) oder des Klitterbuchs synonym gesetzt (Lichtenberg 1992: Vorwort).

6 Der von Friedrich Kittler geprägte Begriff des Aufschreibesystems für das Netzwerk aus Diskursen und Techniken zur Beschreibung des medialen Bruchs 1800/1900 (Kittler 1985) wird heute sehr unterschiedlich verwendet. Wir begreifen unter einem Aufschreibesystem im Bezug auf die praktische Medizin zusammenfassend die Summe der von einem Schreiber oder einer Einrichtung zur Aufzeichnung, Dokumentation und Weitergabe von Beobachtungen eingesetzten Medien. Die Materialität dieser Medien kann Bestandteil sehr unterschiedlicher Schreib- und Drucktechniken sein.

7 Zu den verschiedenen Genres klinischer Aufzeichnungen siehe Hess 2010.

8 Dafür spricht die zeitliche Koinzidenz: Antoine Deidier war von 1711 bis $1714 \mathrm{dem}$ service médical am Hôpital als médecin adjoint en titre Raymond Vieussens zugeordnet, der als Le Médecin Chef von 1694 bis 1714 den service médical bestritt. Nachdem Deidier 1714 in diese Funktion aufrückt war, wurde 1732 die Funktion des médecin adjoint en titre als regelmäßig zu besetzende Stelle eingerichtet (Dulieu 1983: 573 f.).

9 Sauvages erhielt schließlich in Marsillargues eine Stelle als Stadtarzt (Brockliss/Jones 1997: 661, 747).

10 Damit sollen die meist nur kurze Zeit existierenden klinischen Ausbildungsmodelle von Giambattista da Monte (1498-1552) in Padua, Theophrast Renaudot (1586-1653) in Paris, Willem van der Straaten (1593-1681) in Utrecht und sowie der im Leidener Caecilienhospital von Ottho Heurnius (1577-1625) und Franciscus de le Boe (1614-1672) angebotene Unterricht nicht unterschlagen werden.

11 Matiere: 20 (ADH, $10 \mathrm{~F} 53)$.

12 Ebd.: 82.

13 Ebd.

14 Ebd.

15 Ebd.: 7, 55.

16 Ebd.

17 Vgl. Eintrag Phrenesie in ebd.: 2, 31. Auch für eine Observation curiouse (ebd.: 50) wurde sicherlich nicht vorab Platz vorgehalten. 
18 Vgl. Anm. 2.

19 Leider wissen wir nicht, ob mit „les Allemands" eine dezidierte Richtung oder bestimmte Autoren gemeint sind. Die Übersetzungen aus dem Französischen und Lateinischen sind, wenn nicht anders angegeben, von $\mathrm{VH}$.

20 Livre: 14 (ADH, 10 F 51), Sauvages 1763: 300.

21 Meckel 1755, Livre: 251 (ADH, 10 F 51).

22 Eine vollständige Identifizierung der im Livre exzerpierten Observationes und ihr Abgleich mit der Nosologie méthodique steht noch aus.

23 Livre: 54 (ADH, 10 F 51).

24 Ebd.: 47, 57. Die Seitenzahlen beziehen sich auf jene Seiten, auf denen sich eine der unregelmäßig angegebenen Datumsangaben finden lassen.

25 „[C]ollica est, \& ad colicam spasmodicam Scheuchzeri proxime accedit“ (ebd.: 54, Linné 1737: 67-69).

26 Livre: 54 (ADH, 10 F 51).

27 Ebd.: 36.

28 ADH, 10 F 51.

29 Zur Biografie siehe Graetzer 1889: 74.

30 Zur damaligen Verwendung des Begriff „Volkskrankheiten“ siehe den Eintrag in: Johann Heinrich Zedler, 1737. Grosses vollständiges Universal-Lexicon Aller Wissenschafften und Künste. Halle/Leipzig: Zedler, Bd. 16: 432.

31 Zur Beschreibung dieses Genres siehe Hess/Schlegelmilch 2013.

32 Ausführlicher dargestellt ist diese Schreibtechnik für die Société Royale de Médecine bei Mendelsohn 2011.

33 Im Original: „Asthenia generalis, dolor capitis \& praecordium, diarrhoea serosa biliosa, agrypnia, delirium in quibusquam rabiosum et acrophobicum, secunda die sitis importuna, vomitus \& diarrhoea biliosa ... mors epileptica“.

34 Bei solchen Übernahmen wurden Exzerpte durchaus nochmals dupliziert und an unterschiedlichen Stellen in die Fallsammlung eingefügt (siehe hierzu Hess/Mendelsohn 2010).

35 Vgl. hierzu die Darstellungen zu Beobachtungen und zum Schreiben in der Naturgeschichte der Renaissance in Grafton/Siraisi 1999, Pomata/Siraisi 2005 und Ogilvie 2006. Zum Exemplarischen siehe Willer/Ruchatz/Pethes 2007.

36 Die Einführung der binominalen Nomenklatur wird gerne Linné zugeschrieben. Der Publikation der Systema naturae (1735) ging jedoch ein intensiver Austausch zwischen Sauvages und Linné voraus, bei der dieser den „berühmten Gelehrten“ der Montpellienser Fakultät um die Überlassung seiner Nouvelles classes bat (Berg 1956, zum Briefwechsel siehe Hombres-Firmas 1860 und Grasset 1896).

37 Eine plausible Kritik dieses beliebten Arguments gibt bereits Behrisch 2008.

38 Rühmte sich Linné noch 1733, die Anzahl der Pflanzenarten auf 800 Spezies reduziert zu haben, so führte er in der 12. Auflage der Systema naturae ungefähr 7.700 Pflanzen-, 6.200 Tier- und 500 Mineralienarten auf (Bryk 1953).

39 Rey 2000 (211-213) weist am Beispiel des Wahnsinns darauf hin, dass Sauvages gerade jene Begriffe, die in der Encyclopedie und in anderen Lexika eliminiert wurden, wieder in die Debatte eingeführt hat.

40 Selten referiert Sauvages einzelne Fallgeschichten als Observationes. Ohne dass wir diese Beobachtungen quantifizieren können, so scheint er doch solche Observationes vor allem der eigenen Praxis entnommen zu haben.

41 Francesco Torti belegte seine Behandlungsweise der bösartigen Fieber mit zahlreichen „Historiae mirabiles“, wobei er unter anderem eine Reihe von Observationes unter das Caput einer „Febris subcontinua malignans“ subsummierte. Torti bezeichnete seine Fallbeobachtungen wegen des zwar täglich, aber zu unregelmäßigen Zeiten auftretenden Fiebers durchgängig als Beispiele eines besonders bösartigen „Intermittens tertiana duplex". Sauvages versuchte zwischen diesen beiden zu differenzieren, denn er gab Tortis Fallsammlung nicht nur als Beleg für die Quotidiana deceptiva, sondern auch als Grundlage der Tertiana subcontinua an. Wie im oben erörterten Beispiel der Colica spasmodica differenzierte Sauvages die von verschiedenen Autoren gleich betitelten Beobachtungen und ordnete sie unterschiedlichen Systemstellen zu.

42 Dieser Vorgang erfolgte im strikten Gegensatz zu modernen Krankheitsklassifikationen, die 
von Auflage zu Auflage die Zahl der definierten Krankheiten und disorders enorm vermehren.

43 Ein Beleg für diese mehrzeitige Aufschreibepraxis sind die unter der Überschrift „Histoire des mauvais fievres“ versammelten Fallgeschichten aus den Jahren 1742 bis 1761, die Sauvages aus der eigenen Praxis zusammentrug (Livre: 21 f. (ADH, 10 F 51). Diese zwei Seiten stellen nicht nur eines der wenigen Relikte der traditionellen Loci communes dar, sondern belegen anschaulich, dass Sauvages eine Art von Krankenjournal geführt haben dürfte.

44 Unabhängig davon, ob die Medizin nun von Krankheitsentitäten oder disorders spricht, werden in der rezenten Klinik die klassifizierten Krankheiten gerne als substantielle Unterscheidungen betrachtet.

45 Johann Heinrich Zedler, 1737. Leipyria. Grosses vollständiges Universal-Lexicon Aller Wissenschafften und Künste. Halle/Leipzig: Zedler, Bd. 16: 1652.

\section{Literatur}

Baillou, Guillaume de, 1640. Epidemiorum et Ephemeridum Libri Duo. Paris: Quesnel.

Baron, Sabrina Alcorn/Lindquist, Eric N./Shevlin, Eleanor, Hg., 2007. Agent of Change. Print Culture Studies after Elizabeth L. Eisenstein. Amherst [u. a.]: University of Massachusetts Press.

Behrisch, Lars, 2008. Zu viele Informationen! Die Aggregierung des Wissens in der Frühen Neuzeit. In: Arndt Brendecke, Markus Friedrich und Susanne Friedrich, Hg., Information in der Frühen Neuzeit. Status, Bestände, Strategien. Berlin: LIT Verlag, 455-473.

Berg, Fredrik, 1956. Linné et Sauvages. Les Rapports entre leurs Systèmes nosologiques. Lychnos, 16, 31-54.

Berghoff, Emanuel, 1947. Entwicklungsgeschichte des Krankheitsbegriffes. Wien: Maudrich.

Beukers, Harm, 1989. Clinical Teaching in Leiden from its Beginning until the End of the Eighteenth Century. In: Harm Beukers und John Michael Henderson Moll, Hg., Clinical Teaching, Past and Present. Amsterdam [u. a.]: Rodopi, 139-152.

Blair, Ann, 1992. Humanist Methods in Natural Philosophy. The Commonplace Book. Journal of the History of Ideas, 53, 541-551.

Blair, Ann, 2004. Note Taking as an Art of Transmission. Critical Inquiry, 31, 85-107.

Blair, Ann, 2010a. The Rise of Note-Taking in Early Modern Europe. Intellectual History Review, 20, 303-316.

Blair, Ann, 2010b. Too Much to Know. Managing Scholarly Information before the Modern Age. New Haven: Yale University Press.

Bowker, Geoffrey C./Star, Susan Leigh, 1999. Sorting Things Out. Classification and its Consequences. Cambridge, Mass./London: MIT Press.

Brockliss, Laurence/Jones, Colin, 1997. The Medical World of Early Modern France. Oxford, NY: Clarendon.

Bryk, Felix, 1953. Linné und die Species Plantarum. Taxon, 2, 63-73.

Campi, Emidio/De Angelis, Simone/Goeing, Anja-Silvia, Hg., 2008. Scholary Knowledge. Textbooks in Early Modern Europe. Genf: Librairie Droz.

Cooper, Rachel, 2004. What Is Wrong with the DSM? History of Psychiatry, 15, 5-25.

Daston, Lorraine, 2011. The Empire of Observation, 1600-1800. In: Lorraine Daston und Elizabeth Lunbeck, Hg., Histories of Scientific Observation. Chicago/London: The University of Chicago Press, 81-113.

Daston, Lorraine/Lunbeck, Elizabeth, Hg., 2011. Histories of Scientific Observation. Chicago/ London: The University of Chicago Press.

Dulieu, Louis, 1983. La Médecine Á Montpellier. Bd. 3. Avignon: Les Presses Universelles.

Faber, Knud, 1930. Nosography. The Evolution of Clinical Medicine in Modern Times. New York: Paul B. Hoeber.

Foreest, Pieter van, 1584. Observationum et Curationum Medicinalium de Febribus Ephemeris et Continuis Libri Duo. Antwerpen: C. Plantinus.

Foucar, Elliott, 2001. Classification in Anatomic Pathology. Pathology Patterns Reviews, 116, Suppl. $1,5-20$. 
Graetzer, J[onas], 1889. Lebensbilder hervorragender schlesischer Ärzte aus den letzten vier Jahrhunderten. Breslau: Schottlaender.

Grafton, Anthony T./Siraisi, Nancy G., Hg., 1999. Natural Particulars. Nature and the Disciplines in Renaissance Europe. Cambridge, Mass./London: MIT Press.

Grasset, Joseph, 1896. Le Médecin de l'Amour au Temps de Marivaux : Étude sur Boissier de Sauvages d'après des Documents Inédits. Montpellier: C. Coulet.

Hahn, [Johann Gottfried], 1757. Description d'une Epidémie qui a Régné Il y a Quelques Années à Brewflaw. Recueil pérodique d'Observations de Médecine, Chirurgie, Pharmacie, 7, 452-463.

te Heesen, Anke, 2000. Boxes in Nature. Studies in History and Philosophy of Science, 31, 381-403.

te Heesen, Anke, 2005a. Accounting for the Natural World. Double-Entry Bookkeeping in the Field. In: Londa Schiebinger und Claudia Swan, Hg., Colonial Botany. Science, Commerce, and Politics in the Early Modern World. Philadelphia: University of Pennsylvania Press, 237251.

te Heesen, Anke, 2005b. The Notebook. A Paper-Technology. In: Bruno Latour und Peter Weibel, Hg., Making Things Public. Atmospheres of Democracy. Cambridge, Mass./London: MIT Press, 582-589.

Hess, Volker, 1993. Von der semiotischen zur diagnostischen Medizin. Die Entstehung der klinischen Methode zwischen 1750 und 1850. Husum: Matthiesen.

Hess, Volker, 2010. Formalisierte Beobachtung. Die Genese der modernen Krankenakte am Beispiel der Berliner und Pariser Medizin (1725-1830). Medizinhistorisches Journal, 45, 293 340.

Hess, Volker/Mendelsohn, J. Andrew, 2010. Case and Series. Medical Knowledge and Paper Technology, 1600-1900. History of Science, 48, 287-314.

Hess, Volker/Schlegelmilch, Sabine, 2013. Praxistagebücher als Quelle und Aufschreibesystem. In: Martin Dinges, Kay Jankrift, Michael Stolberg und Sabine Schlegelmilch, Hg., In the Doctor's Office. Medical Practice in 17th- to 19th-Century Europe. Amsterdam: Rodopi, in Vorbereitung.

Hoffmann, Christoph, 2010. Schreiben als Verfahren der Forschung. In: Michael Gamper, Hg., Experiment und Literatur. Themen, Methoden, Theorien. Göttingen: Wallstein, 181-207.

Hombres-Firmas, Charles Louis Regis, Hg., 1860. Lettres Inédites de Linné à Boissier de la Croix de Sauvages. Alais: A. Veirun.

Johns, Adrian, 1998. The Nature of the Book. Print and Knowledge in the Making. Chicago/London: University of Chicago Press.

Jones, Colin, 1982. Charity and Bienfaisance. The Treatment of the Poor in the Montpellier Region 1740-1815. Cambridge: Cambridge University Press.

Jones, Colin, 1989. The Charitable Imperative. Hospitals and Nursing in Ancien Régime and Revolutionary France. London/New York: Routledge.

Juncker, Johann, 1736. Conspectus Pathologia ad Dogmata Stahliana Praecipue Adornatae et Semeiologiae Potissimum Hippocratico-Galenciae in Forma Tabularum Repraesentatus. Magdeburg: Impensis Orphanotrophei.

Karenberg, Axel, 1997. Lernen am Bett der Kranken. Zur Typologie der frühen Universitätskliniken in Deutschland (1760-1840). Hürtgenwald: Pressler Verlag.

King, Lester S., 1966. Boissier de Sauvages and 18th Century Nosology. Bulletin of the History of Medicine, 60, 43-51.

Kittler, Friedrich A., 1985. Aufschreibesysteme 1800, 1900. München: Fink.

Krämer, Fabian, 2013. Ein papiernes Archiv für alles jemals Geschriebene. Ulisse Aldrovandis Pandechion Epistemonicon. NTM. Zeitschrift für Geschichte der Wissenschaften, Technik und Medizin, N. S., 21, Heft 1.

Krämer, Sybille, 1988. Symbolische Maschinen. Die Idee der Formalisierung in geschichtlichem Abriß. Darmstadt: Wissenschaftliche Buchgesellschaft.

Lenfant, M. H/Loeb, A./Peuvrel, P./Sauvage, M., 2001. Clinical Coding in Oncology. Bulletin $d u$ Cancer, 88, 209-218.

Lichtenberg, Georg Christoph, 1992. Noctes. Ein Notizbuch. Faksimile mit einem Nachwort und Erläuterungen. Herausgegeben von Ulrich Joost. Göttingen: Wallstein Verlag.

Linné, Carl, 1737. Flora lapponica Exhibens Plantas per Lapponiam crescentes, Secundum Systema sexuale collectas in Itinere impensis Soc. Reg. Litter. et Scient. Sueciae a 1732 Institutio: Additis Synonymis et Locis natalibus Omnium, Descriptionibus et Figuris rariorum, Viribus medicatis et Oeconomicis plurimarum. Amsterdam: Schouten. 
Manget, Johann Jacob, 1695. Bibliotheca Medica Practica, Sive Rerum Medicarum Thesaurus Cumulatissimus. Bd. 4. Genf: Chouet.

Manget, Johann Jacob, 1698. Bibliotheca Medico-Practica sive Rerum Medicarum Thesaurus Cumulatissimus Quo Omnes Prorsus Humani Corporis Morbosae Affectiones. Tum Artem Medicam in Genere, Tum Chirurgicam in Specie, Spectantes Ordine Alphabetico Explicantur et per Curationes, Consilia, Observationes, Ae Cadaverum Anatomicas Inspectiones, Tam Hinc Inde Proprias, Quam a Variis, Iisque Praestantissimis Authoribus, Veteribus, E Recentioribus Petitas, Abunde Imo \& Curiose Tractantur. Genf: Joannis Anthonii Chouet.

Martin, Julian, 1990. Sauvage's Nosology. Medical Enlightenment in Montpellier. In: Andrew Cunnigham, Hg., The Medical Enlightenment of the Eighteenth Century. Cambridge: Cambridge University Press, 111-137.

Mayers, Rick/Horwitz, Allan V., 2005. DSM-III and the Revolution in the Classification of Mental Illness. Journal of the History of Behavioral Sciences, 41, 249-267.

Meckel, Johann Friedrich, 1755. Observations sur les Maladies du Coeur. Histoire de l'Académie Royale des Sciences et des Belles-Lettres de Berlin, 556-585.

Meinel, Christoph, 1995. Enzyklopädie der Welt und Verzettelung des Wissens: Aporien der Empirie bei Joachim Jungius. In: Franz M. Eybl, Wolfgang Harms und Hans-Henrik Krummacher, Hg., Enzyklopädien der Frühen Neuzeit. Beiträge zu ihrer Forschung. Tübingen: Max Niemeyer, 162-187.

Mendelsohn, J. Andrew, 2011. The World on a Page. Making a General Observation in the Eighteenth Century. In: Lorraine Daston und Elizabeth Lunbeck, Hg., Histories of Scientific Observation. Chicago/London: The University of Chicago Press, 396-420.

Müller-Wille, Staffan/Scharf, Sara, 2011. Indexing Nature. Carl Linnaeus and his Fact Gathering Strategies. Svenska Linnesällskapets Årsskrift, 31-60.

Ogilvie, Brian W., 2006. The Art of Describing. Natural History in Renaissance Europe. Chicago/ London: The University of Chicago Press.

Pichot, P., 1994. Nosological Models in Psychiatry. The British Journal of Psychiatry, 164, 232-240.

Pomata, Gianna, 2005. Praxis Historalis. The Uses of Historia in Early Modern Medicine. In: Gianna Pomata und Nancy G. Siraisi, Hg., Historia. Empiricism and Erudition in Early Modern Europe. Cambridge, Mass./London: MIT Press, 105-146.

Pomata, Gianna, 2010. Sharing Cases. The Observationes in Early Modern Medicin. Early Science and Medicine, 15, 193-236.

Pomata, Gianna, 2011. Observation Rising. Birth of an Epistemic Genre, 1500-1650. In: Lorraine Daston und Elizabeth Lunbeck, Hg., Histories of Scientific Observation. Chicago/London: The University of Chicago Press, 45-80.

Pomata, Gianna/Siraisi, Nancy G., Hg., 2005. Historia. Empiricism and Erudition in Early Modern Europe. Cambridge, Mass./London: MIT Press.

Primrose, James, 1658. De Febribus Libri Quator. In Quibus Plurimi Veterum, Recentiorum Errores Declarantur et Refelluntur. Rotterdam: Leers.

Probst, Christian, 1972. Der Weg des ärztlichen Erkennens am Krankenbett. Herman Boerhaave und die ältere Wiener Medizinische Schule, Bd.1, 1707-1787. Wiesbaden: Steiner.

Rey, Roselyne, 2000. Naissance et Développement du Vitalisme en France de la Deuxième Moitié Du Xviiie Siècle á la Fin du Premier Empire. Oxford: Voltaire Foundation.

Risse, Guenter B., 1989. Clinical Instruction in Hospitals. The Boerhaavian Tradition in Leyden, Edinburgh, Vienna and Padua. In: Harm Beukers und John Michael Henderson Molls, Hg., Clinical Teaching, Past and Present. Amsterdam [u. a.]: Rodopi, 1-19.

Sauvages, François Boissier de la Croix, 1731. Nouvelles Classes de Maladies, qui dans un Ordre Semblable à celui de Botanistes, Comprennent les Especes E les Genres de Toutes les Maladies, avec öleurs Signes E leurs Indications. Avignon: B. d'Avanville.

Sauvages, François Boissier de la Croix, 1739. Pathologia Methodica, seu de Cognoscendis Morbis. Montpellier: Ioannis Martel.

Sauvages, François Boissier de la Croix, 1759. Pathologia Methodica, seu de Cognocendis Morbis. Lyon: Petri Bruyset.

Sauvages, François Boissier de la Croix, 1763. Nosologia Methodica Sistens Morborum Classes, Genera et Species, Juxta Sydenhami Mentem et Botanicorum Ordinem. 4 Bde. Amsterdam: Gebrüder de Tournes.

Sauvages, François Boissier de la Croix, 1772. Nosologie Méthodique ou Distributions des Maladies en Classe, en Genres et en Especes. 10 Bde. Lyon: Jean-Marie Bruyset. 
Scheuchzer, Johann Jakob, 1723. Uresiphoites Helveticus, sive Itinera per Helvetiae Alpinas Regiones Facta Annis Mdccii, Mdcciii, Mdcciv, Mdccv, Mdccvi, Mdccvi, Mdccvii, Mdccviii, Mdccix, Mdccx, Mdccxi. Plurimis Tabulis Aeneis Illustrata. Leiden: Pieter van der Aa.

Sennert, Daniel, 1676. Operum in sex Tomos Divisorum. Tomus Quintus-[Sextus]. Lyon: JeanAntoine Huguetan.

Stolberg, Michael, 2007. Formen und Funktionen medizinischer Fallberichte in der Frühen Neuzeit (1500-1800). In: Johannes Süßmann, Susanne Scholz und Gisela Engel, Hg., Fallstudien. Theorie - Geschichte - Methode. Berlin: Trafo, 81-95.

Stolberg, Michael, 2013. Medizinische Loci Communes. Formen und Funktionen einer ärztlichen Aufzeichnungspraxis im 16. und 17. Jahrhundert. NTM. Zeitschrift für Geschichte der Wissenschaften, Technik und Medizin, N. S., 21, Heft 1.

Waquet, Françoise, Hg., 2000. Mapping the World of Learning. The Polyhistor of Daniel Georg Morhof. Wiesbaden: Harrassowitz.

Wieland, Wolfgang, 1975. Diagnose. Überlegungen zur Medizintheorie. Berlin/New York: Walter de Gruyter.

Williams, Elizabeth Ann, 2003. A Cultural History of Medical Vitalism in Enlightenment Montpellier. Aldershot [u. a.] Ashgate.

Willer, Stefan/Ruchatz, Jens/Pethes, Nicolas, 2007. Zur Systematik des Beispiels. In: Jens Ruchatz, Stefan Willer und Nicolas Pethes, Hg., Das Beispiel. Epistemologie des Exemplarischen. Berlin: Kulturverlag Kadmos, 7-59.

Yeo, Richard, 2007. Between Memory and Paperbooks. Baconianism and Natural History in Seventeenth-Century in England. History of Science, 45, 1-46.

Zedelmaier, Helmut, 1992. Bibliotheca Universalis und Bibliotheca Selecta. Das Problem der Ordnung des gelehrten Wissens in der Frühen Neuzeit. Köln [u. a.]: Böhlau.

Zedelmaier, Helmut, 2009. Gelehrtes Wissensmanagement in der Frühen Neuzeit. In: Helmut Neuhaus, Hg., Die Frühe Neuzeit als Epoche. München: Oldenbourg Wissenschaftsverlag, $77-89$.

\section{Volker Hess}

Institut für Geschichte der Medizin

Charité Universitätsmedizin Berlin

Ziegelstraße 10

10117 Berlin

Deutschland

E-Mail: volker.hess@charite.de

Andrew Mendelsohn

School of History

Queen Mary, University of London

London

E1 4NS

UK

E-Mail: a.mendelsohn@qmul.ac.uk 